This is a post-print of the following article: Andric, Predrag; Curtin, W. A. Journal Of The Mechanics And Physics Of Solids $2017,23$. 315-337.. The formal publication is available at http://dx.doi.org/10.1016/j.jmps.2017.06.006 ( ) 2017. This manuscript version is made available under the CC-BY-NC-ND 4.0 license http://creativecommons.org/licenses/by-nc-nd/4.0/

\title{
New Theory for Mode I Crack-tip Dislocation Emission
}

\author{
Predrag Andric, W.A. Curtin \\ Laboratory for Multiscale Mechanics Modeling \\ EPFL \\ CH-1015 Lausanne, Switzerland
}

\begin{abstract}
A material is intrinsically ductile under Mode I loading when the critical stress intensity $K_{I e}$ for dislocation emission is lower than the critical stress intensity $K_{I c}$ for cleavage. $K_{I e}$ is usually evaluated using the approximate Rice theory, which predicts a dependence on the elastic constants and the unstable stacking fault energy $\gamma_{\text {usf }}$ for slip along the plane of dislocation emission. Here, atomistic simulations across a wide range of fcc metals show that $K_{I e}$ is systematically larger (10-30\%) than predicted. However, the critical (crack tip) shear displacement is up to $40 \%$ smaller than predicted. The discrepancy arises because Mode I emission is accompanied by the formation of a surface step that is not considered in the Rice theory. A new theory for Mode I emission is presented based on the ideas that (i) the stress resisting step formation at the crack tip creates "lattice trapping" against dislocation emission such that (ii) emission is due to a mechanical instability at the crack tip. The new theory is formulated using a Peierls-type model, naturally includes the energy to form the step, and reduces to the Rice theory (no trapping) when the step energy is small. The new theory predicts a higher $K_{I e}$ at a smaller critical shear displacement, rationalizing deviations of simulations from the Rice theory. Specific predictions of $K_{I e}$ for the simulated materials, usually requiring use of the measured critical crack tip shear displacement due to complex material non-linearity, show very good agreement with simulations. An analytic model involving only $\gamma_{\mathrm{usf}}$, the surface energy $\gamma_{\mathrm{s}}$, and anisotropic elastic constants is shown to be quite accurate, serves as a replacement for the analytical Rice theory, and is used to understand differences between Rice theory and simulation in recent literature. The new theory highlights the role of surface steps created by dislocation emission in Mode I, which has implications not only for intrinsic ductility but also for crack tip twinning and fracture due to chemical interactions at the crack tip.
\end{abstract}

Keywords: Cracks, Dislocations, Fracture, Molecular Statics Simulations 
This is a post-print of the following article: Andric, Predrag; Curtin, W. A. Journal Of The Mechanics And Physics Of Solids $2017,, 23$. 315-337.. The formal publication is available at http://dx.doi.org/10.1016/j.jmps.2017.06.006 ( ) 2017. This manuscript version is made available under the CC-BY-NC-ND 4.0 license http://creativecommons.org/licenses/by-nc-nd/4.0/

\section{Introduction}

The heightened need for reduced energy consumption across many industries drives the search for improvement structural performance and reliability of materials. High structural performance and reliability are achieved with increased fracture toughness. A fundamental requirement for achieving high fracture toughness in crystalline metals is that a material be intrinsically ductile. A crystalline metal is intrinsically ductile if an atomically sharp crack in a loaded material emits dislocation(s) and blunts rather than cleaving and remaining sharp. Specifically, if the Mode I stress intensity factor for emission $K_{I e}$ is smaller than the Mode I stress intensity factor for cleavage $K_{I c}[1]$, then the material will emit dislocations, blunt, and eventually fail by mechanisms that absorb considerable energy. While the overall fracture toughness is governed by many larger-scale factors, materials that are intrinsically brittle, i.e. $K_{I c}<K_{I e}$, often have insufficient toughness for failure-critical applications. Dislocation emission from the crack tip is thus a necessary precursor to crack tip blunting and toughening, while also representing one of the classical problems in the mechanics of materials.

In light of its practical importance, a number of continuum mechanics models have been introduced to predict crack tip dislocation emission [2-6]. The most widely-used model is that by Rice, which is based on a cohesive model for slip displacement ahead of the crack [7]. Under Mode II (in-plane shear) loading, Rice showed that emission is controlled by an energy criterion involving the unstable stacking fault (USF) energy $\gamma_{u s f}$. The USF is a saddle point on the generalized stacking fault energy surface associated with relative shear displacements of two rigid blocks of material. Under Mode I loading, the Mode II analysis does not apply directly, but Rice postulated that, at the point of emission, the slip profile along the slip plane is the same as that in Mode II. This yields a dependence of $K_{I e}$ on $\gamma_{u s f}$ and on the orientation of the slip plane relative to the crack front.

Molecular statics and dynamics simulations provided a means by which to validate the Rice model. Early simulations [8,9] showed that the Rice criterion gives accurate predictions for $K_{I I e}$ under Mode II loading, where the crack plane is coplanar with the slip plane. However, results for Mode I loading showed varying levels of agreement from material to material. It was recognized that deviations from the Rice criterion could be due to the creation of a surface step (surface ledge) during the Mode I nucleation process. Several authors thus tried to incorporate the additional energy of the surface step [10-13] into a Ricetype analysis, but usually for one particular material with some approximations, and without 
This is a post-print of the following article: Andric, Predrag; Curtin, W. A. Journal Of The Mechanics And Physics Of Solids $2017,23$. 315-337.. The formal publication is available at http://dx.doi.org/10.1016/j.jmps.2017.06.006 @ 2017. This manuscript version is made available under the CC-BY-NC-ND 4.0 license http://creativecommons.org/licenses/by-nc-nd/4.0/

achieving significantly better results. In all of these models, the key Rice concept was maintained: the unstable stacking fault energy controls the dislocation nucleation with emission occurring when the slip displacement reaches the displacement corresponding to the unstable stacking fault displacement. Schoeck (2003) [14] considered a related continuum model, but with creation of a step introduced through an additional constant force acting at the crack tip. The resulting energy functional was then solved approximately using a variational method to obtain the slip distribution along the slip plane, and results were shown for simplified slip energy models. One conclusion of the Schoeck analysis is that, for low step energy, dislocation emission could occur below the Rice prediction, which is not generally supported by simulations in Mode II or Mode I for atomically sharp cracks and is difficult to rationalize physically. Schoeck was pursuing a valuable path that is echoed here, but with an approximate model. Zamora et al. [33] recently proposed a continuum approach that included extra energy for step formation near the crack tip and proposed a method for computing the step energy contribution, but they presented limited results for a specific system where the role of surface step creation was not clearly identified.

Here, we approach the Mode I emission problem as a mechanical instability governed by a critical crack tip displacement. We show that, in contradiction to a key assumption in the Rice theory, the energy change at the crack tip due to relative slip is monotonically increasing with crack tip displacement, due to the energy cost of creating the step. Thus, the Rice theory simply cannot apply: there is no saddle point in the energy versus slip. We then develop a model that assumes all non-linear response to occur at the crack tip to demonstrate that, in the presence of the step, there exists a critical crack tip displacement at which mechanical instability occurs, i.e. the driving stress at the crack tip due to the applied field can no longer be balanced by the restoring stresses that resist step formation. The simple model rationalizes simulation trends and provides analytic results. We apply the model to 17 different fcc materials where, due to material non-linearities, we use the measured critical crack tip displacement in the theory and then predict $K_{I e}$ in very good agreement with the simulated values. The new theory captures all key aspects of the Mode I dislocation nucleation process, resolving the discrepancies of the Rice theory. A simplified analytic model is then presented that involves only easily-computable material properties yet shows excellent agreement with the simulations. The simplified model is also used to rectify previous discrepancies between Rice theory and atomistic simulations in other materials. 
This is a post-print of the following article: Andric, Predrag; Curtin, W. A. Journal Of The Mechanics And Physics Of Solids $2017,23$. 315-337.. The formal publication is available at http://dx.doi.org/10.1016/j.jmps.2017.06.006 ( ) 2017. This manuscript version is made available under the CC-BY-NC-ND 4.0 license http://creativecommons.org/licenses/by-nc-nd/4.0/

The remainder of this paper is organized as follows. In Section II, we give a more detailed exposition of the Rice theory and its predictions for $K_{I e}$ and the critical slip $\Delta_{c}$ for elastically anisotropic materials $[15,16]$. In Section III, we first introduce 17 fcc materials that will be studied, we then carefully validate the Rice model for Mode II loading for these materials, and finally we present results for Mode I loading, with a slip plane inclined at an angle $\theta=70.53^{\circ}$ with respect to the crack plane, and show clear deviations from the Rice theory. In Section IV, we show that the energy change at the crack tip during the emission/slip process has contributions from the surface step energy as well as the stacking fault energy. In section $\mathrm{V}$, we developed the new theory for crack tip dislocation emission based on a mechanical instability at the crack tip. In Section VI, we compare the new theoretical model for dislocation emission to simulations across a wide range of fcc materials. In section VII, we introduce an analytic criteria and show it to be in good agreement with the full model results. Implications of the new model are then discussed and our main results reiterated in Section VIII.

\section{Review of Rice criterion for dislocation emission}

Rice formulated a criterion for crack tip dislocation emission based on the Peierls concept [17]. This concept assumes the existence of a periodic energy functional $\Psi_{g s f}$ that is a function of the relative slip $\Delta$ between two rigid crystal blocks. The energy $\Psi_{g s f}$ is the socalled generalized stacking fault energy (GSF energy) evaluated at relative slip $\Delta$, with $0 \leq$ $\Delta \leq b$ where $\mathrm{b}$ is the Burgers vector of the emitted dislocation. For fcc materials, the focus of the work here, the emitted dislocation is a partial dislocation with Burgers vector $b_{p}$. The emission of the partial dislocation leaves behind a stable stacking fault as the dislocation glides away from the crack tip. A typical GSF energy function is shown in Figure 1, obtained from an atomistic simulation at $\mathrm{T}=0 \mathrm{~K}$ for an fcc Ni EAM potential [19]. The unstable stacking fault is at $\Delta \sim b_{p} / 2$ and the stable stacking fault $\gamma_{s s f}$ is at $\Delta=b_{p}$. The GSF curve is a material property, independent of any crack geometry or mode of loading in a crack problem.

Rice formulated the problem of dislocation emission ahead of a semi-infinite crack under pure Mode II loading as a Mode II cohesive zone problem where the GSF energy defines the (continuum) cohesive response ahead of the crack tip via the material shear resistance as $\tau=d \Psi_{g s f}(\Delta) / d \Delta$. The cohesive model eliminates the elastic stress singularity at the crack tip in analogy to a cohesive/bridging/Dugdale zone in the Mode I fracture problem. Since the GSF curve includes the elastic response of the material that is already 
This is a post-print of the following article: Andric, Predrag; Curtin, W. A. Journal Of The Mechanics And Physics Of Solids $2017,23$. 315-337.. The formal publication is available at http://dx.doi.org/10.1016/j.jmps.2017.06.006 @ 2017. This manuscript version is made available under the CC-BY-NC-ND 4.0 license http://creativecommons.org/licenses/by-nc-nd/4.0/

contained in the elastic field, Rice introduced the displacement discontinuity $\delta$, which is the inelastic slip, i.e. the additional slip beyond that predicted by elasticity. He then showed that $\delta=\Delta-h \tau(\Delta) / \mu$, where $h$ is the atomic inter-planar spacing and $\mu$ is the shear modulus (along the plane of sliding). Rice then introduced the inelastic potential $\Phi(\delta)$ for the energy associated with the inelastic slip, given by

$$
\Phi(\delta)=\Psi_{g s f}(\Delta)-h \tau^{2}(\Delta) / 2 \mu,
$$

Within the framework of the cohesive model, the path-independent J-integral can be applied. In isotropic elasticity, the J-integral along a contour far from the crack is $J_{\text {far }}=(1-v) K_{I I}^{2} / 2 \mu=G$ where $\mathrm{G}$ is the macroscopic energy release rate. The J-integral along a crack face contour around the cohesive zone is given by

$$
J=-\int_{0}^{\infty} \tau \frac{\partial \delta}{\partial x} d x=\int_{0}^{\delta_{t i p}} \tau(\delta) d \delta \equiv \Phi\left(\delta_{t i p}\right),
$$

Path independence of the J-integral leads to the result

$$
G \equiv(1-v) K_{I I}^{2} / 2 \mu=\Phi\left(\delta_{\text {tip }}\right)
$$

An incipient dislocation (partial slip distribution along the slip plane) loses stability when the slip discontinuity at the crack tip reaches the critical unstable stacking fault position, $\delta_{c}^{t i p}=\Delta_{u s f}$, at which point $\delta$ and $\Delta$ are equal, $\delta_{c}^{t i p}=\Delta_{c}^{t i p}=\Delta_{u s f}$. At this point, the inelastic slip energy is a maximum and equal to $\gamma_{u s f}$, and so $G$ is also a maximum and corresponds to the point of dislocation emission. Within isotropic elasticity, the corresponding critical stress intensity factor for dislocation emission is then

$$
K_{\text {IIe }}=\sqrt{2 \mu \gamma_{u s f} /(1-v)} \text {. }
$$

For Mode I loading, where the slip plane is inclined at some angle $\theta$ to the crack 115 plane, there is no exact solution. The crack tip geometry is not self-similar and the J-integral concept does not apply. Rice proposed that, at the point of emission, the distribution of shear slip along the slip plane is the same in Modes I and II. This allows the result for Mode II to be used to estimate emission for Mode I by computing the effective Mode II loading along the slip plane, leading to

$$
K_{I e}=\sqrt{2 \mu \gamma_{u s f} /(1-v)} / \cos ^{2}(\theta / 2) \sin (\theta / 2)
$$


This is a post-print of the following article: Andric, Predrag; Curtin, W. A. Journal Of The Mechanics And Physics Of Solids $2017,23$. 315-337.. The formal publication is available at http://dx.doi.org/10.1016/j.jmps.2017.06.006 @ 2017. This manuscript version is made available under the CC-BY-NC-ND 4.0 license http://creativecommons.org/licenses/by-nc-nd/4.0/

120 with emission again occurring when $\Delta_{c}^{t i p}=\Delta_{u s f}$. The isotropic results of Eqs. 4 and 5 were generalized to full anisotropic elasticity [15] with the result

$$
\begin{aligned}
& K_{I I e}=\sqrt{\gamma_{u s f} o(\theta, \phi)} \\
& K_{I e}=\sqrt{\gamma_{u s f} o(\theta, \phi)} / F_{12}(\theta)
\end{aligned}
$$

where $o(\theta, \phi)$ is function of the slip plane angle $\theta$ and the angle $\varphi$ between the dislocation Burgers vector and the crack front direction in the slip plane (see Appendix A) and $F_{12}(\theta)$ is related to the angular distribution of shear stress at the crack tip.

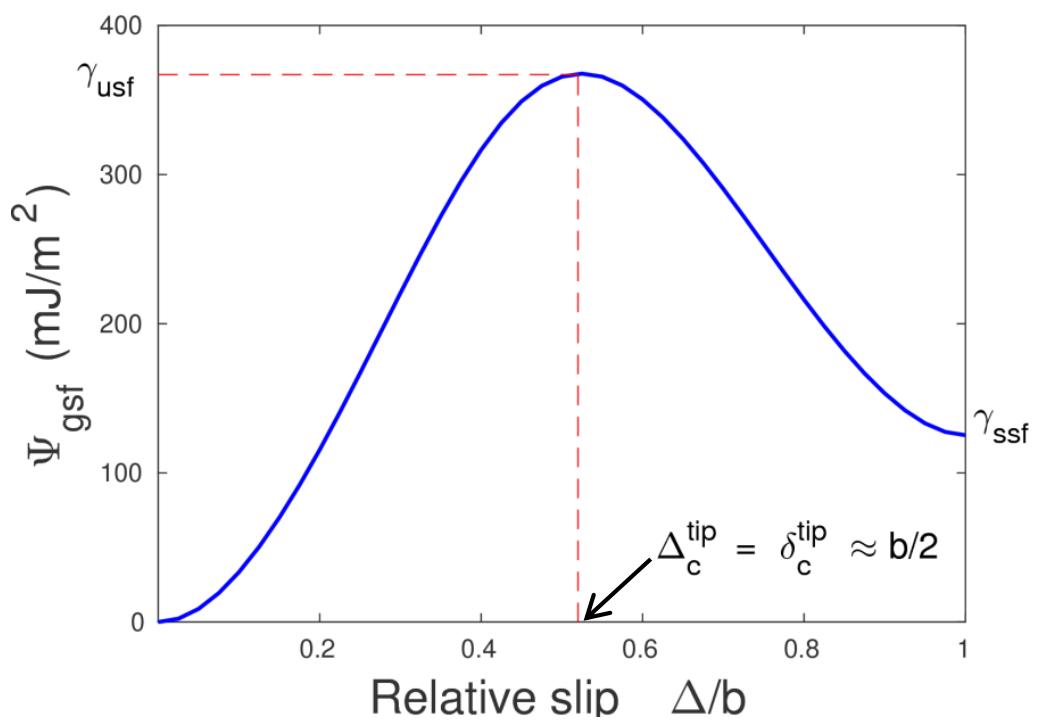

Figure 1: Generalized stacking fault energy computed in Nickel at $\mathrm{T}=0 \mathrm{~K}$.

\section{Rice theory versus atomistic simulations}

\section{III.1 Materials studied}

The crack tip dislocation emission is analyzed for 17 different fcc materials. To describe Aluminum interatomic interactions we use two different EAM potentials developed by Mishin et al. [19] and Ercolessi and Adams [20]. Mishin EAM potentials were also used to describe Ni [19] and Copper [21] while the Adams et al. [22] EAM potentials were used to describe Gold, Silver and Palladium. A set of effective alloy potentials, labeled Cr10-Cr100, were derived using a homogenization procedure [24] based on an Fe-Ni-Cr EAM ternary system developed by Bonny [23]. The homogenization procedure enables the creation of a set 
This is a post-print of the following article: Andric, Predrag; Curtin, W. A. Journal Of The Mechanics And Physics Of Solids $2017,, 23$. 315-337.. The formal publication is available at http://dx.doi.org/10.1016/j.jmps.2017.06.006 @ 2017. This manuscript version is made available under the CC-BY-NC-ND 4.0 license http://creativecommons.org/licenses/by-nc-nd/4.0/

of single-atom materials with continuous property variations by continuous change of the underlying alloy composition. The Cr10-Cr100 materials are a discrete set of materials of

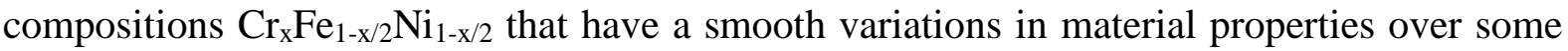
range. It so happens that these materials have nearly constant (111) surface energies while the stacking fault energies, GSF curves, slip $\Delta_{u s f}$ at the unstable stacking fault, and anisotropic elastic properties vary significantly. The relevant properties for each material, as computed in atomistic simulations using the above EAM potentials, are presented in Appendix A.

\section{III.2 Mode II loading}

To validate the Rice criterion for crack tip dislocation emission in Mode II, we perform molecular statics simulations using the molecular dynamics code LAMMPS [25] as follows. We simulate a semi-infinite crack under plane strain loading conditions, with the crack in the $\mathrm{x}-\mathrm{z}$ plane, and crack tip at $\mathrm{y}=0$ with the crack line along $\mathrm{z}$. The simulation cell has dimensions of approximately $150 \times 150 \times 1 \mathrm{~nm}$ with periodic boundary conditions along the crack line direction (see Figure 2). Because we simulate emission at $T=0 \mathrm{~K}$ and in homogeneous atomic systems, results are independent of the sample length along the crack line direction. The crack is loaded incrementally by applying increments of anisotropic displacement based on linear elastic fracture mechanics as

$$
\begin{aligned}
& \Delta u_{x}=\Delta K_{I I} \sqrt{\frac{2 r}{\pi}} \operatorname{Re}\left[\frac{1}{a_{1}-a_{2}}\left(p_{2} \sqrt{\cos \theta+a_{2} \sin \theta}-p_{1} \sqrt{\cos \theta+a_{1} \sin \theta}\right)\right] \\
& \Delta u_{y}=\Delta K_{I I} \sqrt{\frac{2 r}{\pi}} \operatorname{Re}\left[\frac{1}{a_{1}-a_{2}}\left(q_{2} \sqrt{\cos \theta+a_{2} \sin \theta}-q_{1} \sqrt{\cos \theta+a_{1} \sin \theta}\right)\right]
\end{aligned}
$$

where $a_{1}$ and $a_{2}$ are roots of the characteristic equation and $p_{1}, p_{2}, q_{1}$ and $q_{2}$ depend on the material compliance tensor and $a_{1}$ and $a_{2}$; for more details see Appendix A. After each increment of displacement, atoms within $2 r_{c}\left(r_{c}=\right.$ cut-off distance of the interatomic potential) of the outer boundary of the simulation cell (green atoms in Figure 2) are held fixed at the elastic displacement solution and all other atoms are relaxed to minimize the total energy of the system using the conjugate gradient method. The load is incrementally increased in steps of $\Delta K_{I I}=0.001 \mathrm{MPa} \sqrt{\mathrm{m}}$. The simulation is terminated when the first partial dislocation is emitted from the crack tip. 
This is a post-print of the following article: Andric, Predrag; Curtin, W. A. Journal Of The Mechanics And Physics Of Solids $2017,23$. 315-337.. The formal publication is available at http://dx.doi.org/10.1016/j.jmps.2017.06.006 ( ) 2017. This manuscript version is made available under the CC-BY-NC-ND 4.0 license http://creativecommons.org/licenses/by-nc-nd/4.0/
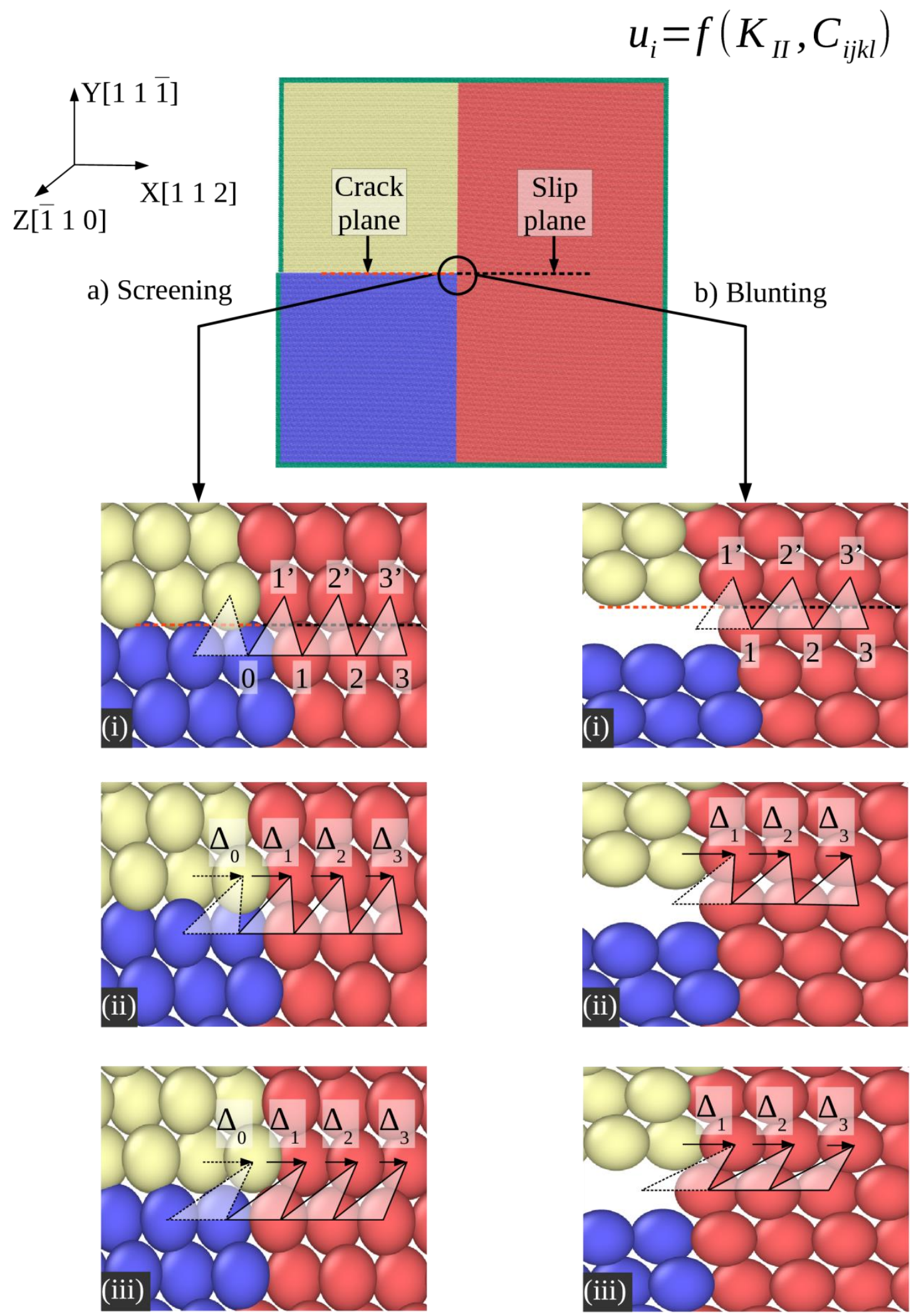

Figure 2: Crack geometry in Mode II loading formed by a) deleting the interatomic interactions between the crack surfaces (yellow and blue atoms, respectively) and denoted as "screening" and b) removing one layer of atoms and then screening the remaining atoms (yellow and blue, respectively) and denoted as "blunting". For both (a) and (b), the crack geometry is shown (i) at zero load, (ii) at the critical load for dislocation emission, and (iii) after dislocation emission. The triangles are the basic structural units through which the shear displacement along the plane of emission are analysed, as indicated. Atoms are visualized using OVITO [26]. 
This is a post-print of the following article: Andric, Predrag; Curtin, W. A. Journal Of The Mechanics And Physics Of Solids $2017,23$. 315-337.. The formal publication is available at http://dx.doi.org/10.1016/j.jmps.2017.06.006 ( ) 2017. This manuscript version is made available under the CC-BY-NC-ND 4.0 license http://creativecommons.org/licenses/by-nc-nd/4.0/

The above description is general. For pure Mode II loading, the fcc crystal is oriented with $X=\left[\begin{array}{lll}1 & 1 & 2\end{array}\right], Y=\left[\begin{array}{lll}1 & 1 & \overline{1}\end{array}\right], Z=\left[\begin{array}{lll}\overline{1} & 1 & 0\end{array}\right]$, creating a crack with a slip plane coplanar with the crack. A Mode II crack in an atomistic simulation cannot be created naturally - the tractionfree surface of the idealized Mode II crack cannot simply be imposed on atoms. We use two approaches to simulate a Mode II crack. In the first approach (Figure 2a), called "screening", we eliminate all inter-atomic forces between atoms on either side of the initial crack surfaces (yellow and blue atoms, respectively). Because realistic interatomic potentials are multi-body potentials, "screening" influences the behaviour of all the atoms at the crack tip, and so deviations from the ideal Mode II crack can arise. In the second approach (Figure 2b), called "blunting", we remove one or more rows of atoms to create crack surfaces. The finite distance between the remaining atoms on either side of the crack behind the crack tip leads to reduced interactions between these atoms, approaching the desired traction-free condition. However, this approach creates a slightly blunted crack, and so deviations from the ideal perfectly sharp Mode II crack can again arise. Here, we consider both "screening" and a combination of "blunting" by removal of one atomic row of atoms plus "screening" of the remaining atoms across the crack surfaces. Simulations show that these two cases give quite similar results for Mode II and also bracket the predictions of the Rice theory.

Figure 3 shows the results for the critical stress intensity factor $K_{I I e}$ and crack tip relative slip at the point of emission, as simulated for 17 different EAM potentials [19-24] and as predicted by the Rice theory. Figure 3a shows that the Rice prediction for the critical stress intensity $K_{I I e}$ for emission is in very good quantitative agreement with the simulation values, although there are larger deviations for the "screening" case for the Cr60 - Cr100 potentials, which have rather asymmetric GSF curves (see below). Overall, the Rice prediction for $K_{I I e}$ is generally between the two simulation results, which is the best possible agreement we could expect given the uncertainty in setting the atomistic crack tip conditions.

Figure $3 b$ shows the crack tip shear displacement just before emission, as measured in the simulations and as predicted by the Rice theory. We measure the shear displacement along the slip plane as the shear deformation of the atomic triangular structural units indicated in Figure 2. The shear displacement of the crack tip structural unit can be measured as the difference in displacement between the atoms marked by $1^{\prime}$ and $0, \Delta_{1}^{\prime}=u_{1}^{\prime}-u_{0}$, or between the atoms marked by $1^{\prime}$ and $1, \Delta_{1}=u_{1}^{\prime}-u_{1}$, where $u_{i}$ is the displacement of an atom $i$ in the [112] slip direction (see initial crack in Figure 2). Under pure shear, these two values are identical, $\Delta_{1}^{\prime}=\Delta_{1}$, and we find only very small differences between $\Delta_{1}$ and $\Delta_{1}{ }^{\prime}$ in 
This is a post-print of the following article: Andric, Predrag; Curtin, W. A. Journal Of The Mechanics And Physics Of Solids $2017,23$. 315-337.. The formal publication is available at http://dx.doi.org/10.1016/j.jmps.2017.06.006 @ 2017. This manuscript version is made available under the CC-BY-NC-ND 4.0 license http://creativecommons.org/licenses/by-nc-nd/4.0/

the simulated Mode II loading, as expected. The shear displacement shown in Figure 3 is $\Delta_{1}$. In the screening case (Figure 2a), the atomic unit immediately behind the crack tip does not fully satisfies the condition of a traction free crack surface and so we also show the shear deformation $\Delta_{0}$ immediately behind the crack tip, and expect $\Delta_{0}$ and $\Delta_{1}$ to bracket the true value. Overall, the Rice prediction is slightly higher than simulation results, but in broad agreement. There are few cases (the Cr70-Cr100 potentials) where the Rice prediction is notably higher than the simulations results, and for the same potentials we find a larger discrepancy in the prediction of the critical $K_{\text {IIe. }}$ A possible reason for this discrepancy is mentioned in Section V.
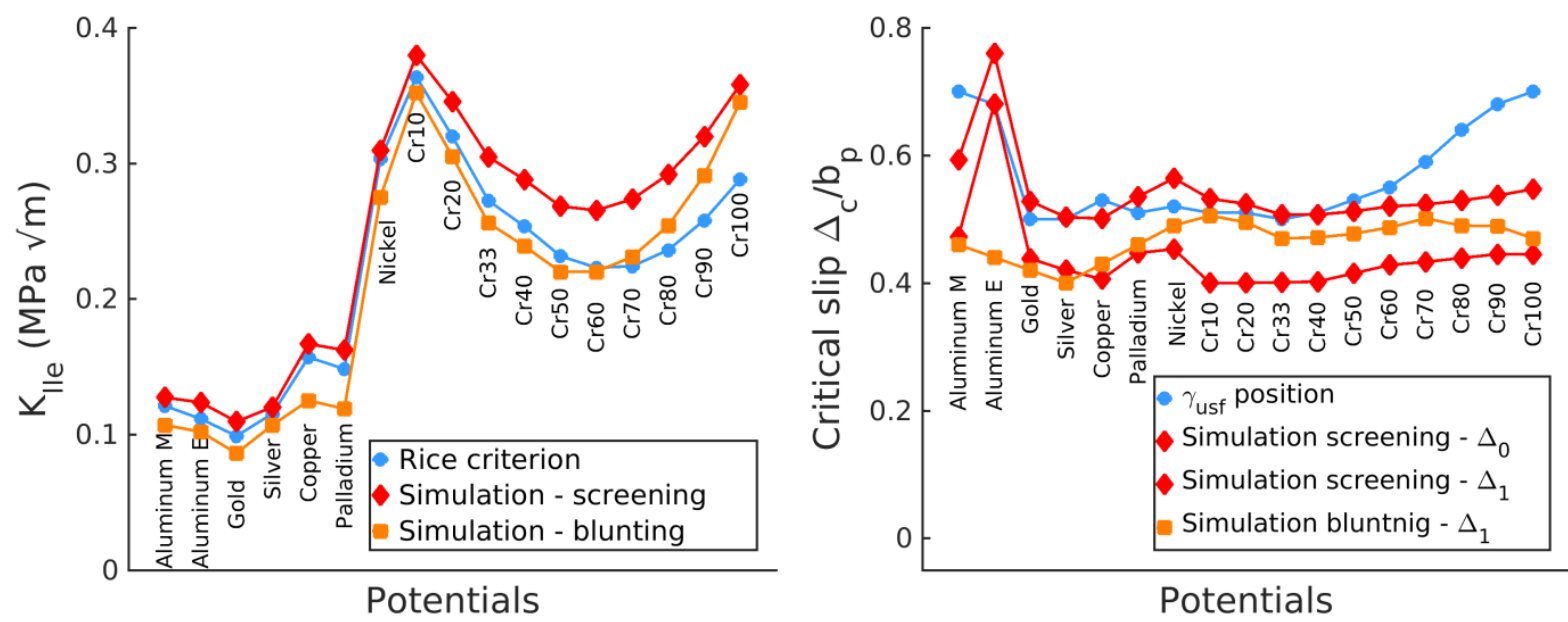

Figure 3: Results of simulations under Mode II loading for both "screening" and "blunting" models: a) Critical stress intensity factor $\mathrm{K}_{\text {IIe }}$ for crack-tip dislocation emission; b) Relative shear displacement of the structural units at the crack tip and, for "screening" only, immediately behind the crack tip. In both (a) and (b), light blue symbols show the predictions of the Rice model.

We conclude that, within the limitations of the ability to simulate an ideal Mode II crack, the Rice model is accurate, qualitatively and quantitatively, in its prediction of dislocation emission under Mode II. Since the Rice theory is intended for Mode II, and since no step is

formed during the emission, the agreement is not surprising. However, it is important to demonstrate this level of agreement between simulation and theory in Mode II because it highlights the clear differences that will arise in Mode I.

\section{III.3 Mode I loading}

We now examine the Rice criterion for crack tip dislocation emission in Mode I loading, performing molecular statics simulations as follows. We simulate semi-infinite crack 
This is a post-print of the following article: Andric, Predrag; Curtin, W. A. Journal Of The Mechanics And Physics Of Solids $2017,23$. 315-337.. The formal publication is available at http://dx.doi.org/10.1016/j.jmps.2017.06.006 @ 2017. This manuscript version is made available under the CC-BY-NC-ND 4.0 license http://creativecommons.org/licenses/by-nc-nd/4.0/

under plane strain loading conditions. We use the same boundary conditions, crack tip position and simulation cell size as in the simulations of Mode II loading. Here, the crystal is oriented with $X=\left[\begin{array}{lll}\overline{1} & 1 & 2\end{array}\right], Y=\left[\begin{array}{lll}1 & 1 & 1\end{array}\right], Z=\left[\begin{array}{lll}\overline{1} & 1 & 0\end{array}\right]$, forming a crack with a slip plane inclined at an angle $\theta=70.53^{\circ}$ to the crack plane (see Figure 4). The crack is, once again, loaded by applying increments of the anisotropic displacement field corresponding to increments of the applied stress intensity factor $\mathrm{K}_{\mathrm{I}}$ given by

$$
\begin{aligned}
& \Delta u_{x}=\Delta K_{I} \sqrt{\frac{2 r}{\pi}} \operatorname{Re}\left[\frac{1}{a_{1}-a_{2}}\left(a_{1} p_{2} \sqrt{\cos \theta+a_{2} \sin \theta}-a_{2} p_{1} \sqrt{\cos \theta+a_{1} \sin \theta}\right)\right] \\
& \Delta u_{y}=\Delta K_{I} \sqrt{\frac{2 r}{\pi}} \operatorname{Re}\left[\frac{1}{a_{1}-a_{2}}\left(a_{1} q_{2} \sqrt{\cos \theta+a_{2} \sin \theta}-a_{2} q_{1} \sqrt{\cos \theta+a_{1} \sin \theta}\right)\right] .
\end{aligned}
$$

After each increment of loading $\Delta K_{I}=0.001 \mathrm{MPa} \sqrt{\mathrm{m}}$, the boundary atoms (within $2 \mathrm{r}_{\mathrm{c}}$ of the outer boundary; green atoms in Figure 4) are held fixed at the elastic displacement solution. All other atoms are then relaxed using the conjugate gradient method and the simulation is terminated when the first partial dislocation is emitted. To prevent crack closure, which will occur at loads below $K_{I c}$, we use (i) "screening" between the yellow and blue atoms (Figure $4 a$ ), or (ii) "blunting" by deletion of three layers of atoms (Figure 4b).

The critical stress intensity factor $K_{I e}$ for emission under Mode I loading is shown in Figure 5a. The Rice theory gives fair quantitative predictions for $K_{I e}$, but is almost always lower than simulations for both "screening" and "blunting". Some differences are large, up to 20-50\% (see also Table 1). Results here are consistent with other results on specific systems scattered through the literature. The second observation is that, as in Mode II, the simulated $K_{I e}$ is usually slightly larger for "screening" as compared to "blunting". There is no clear interpretation of this difference in Rice theory. Beltz and Rice noted that the Rice theory for Mode I does not account for the opening displacement normal to the slip plane, which is expected to reduce the slip energy and thus reduce $K_{I e}$ below the Rice theory; such effects would increase the discrepancy between the theory and simulations. 
This is a post-print of the following article: Andric, Predrag; Curtin, W. A. Journal Of The Mechanics And Physics Of Solids $2017,23$. 315-337.. The formal publication is available at http://dx.doi.org/10.1016/j.jmps.2017.06.006 ( ) 2017. This manuscript version is made available under the CC-BY-NC-ND 4.0 license http://creativecommons.org/licenses/by-nc-nd/4.0/

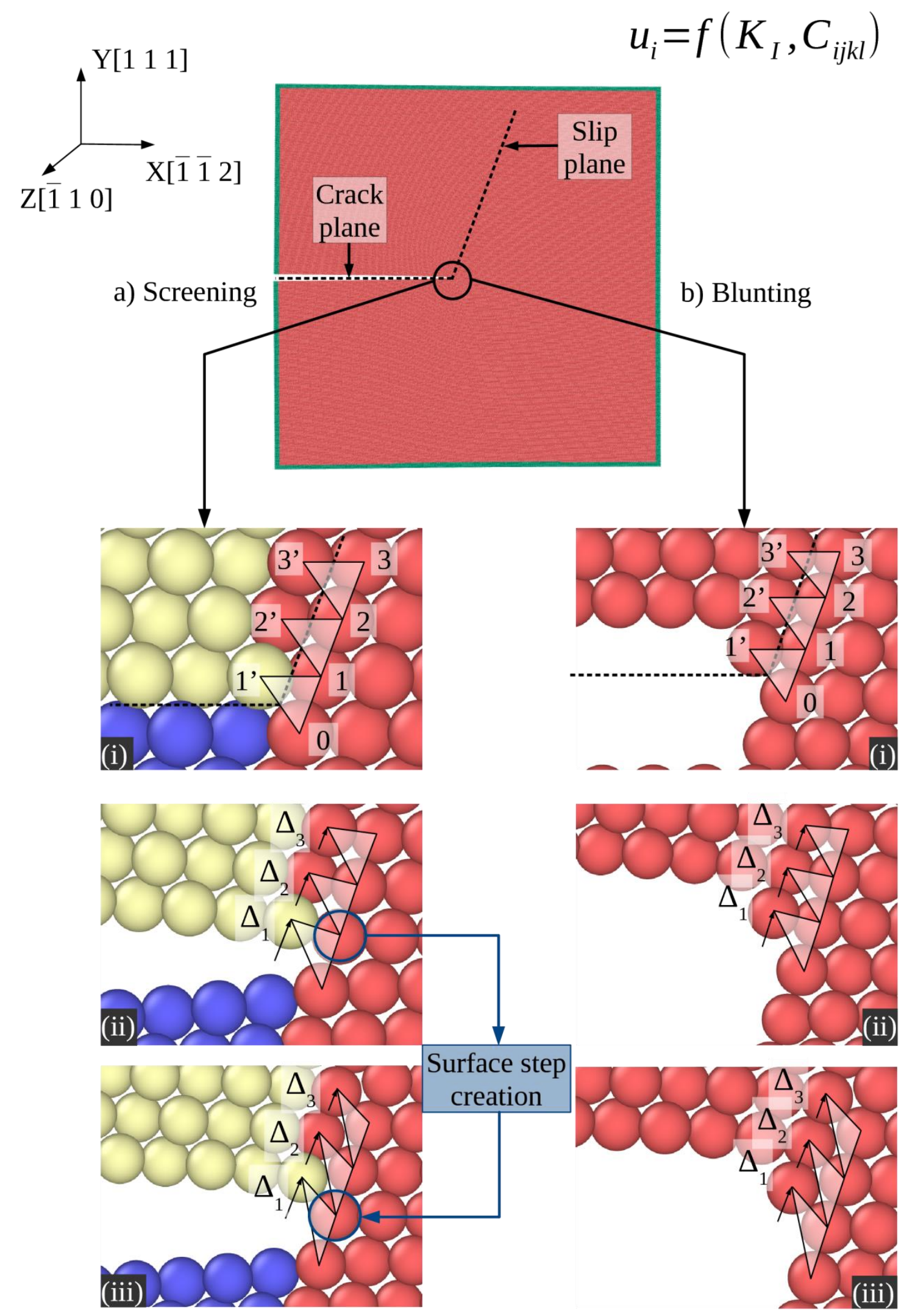

Figure 4: Crack geometry in Mode I loading formed by a) deleting the interatomic interactions between the crack surfaces (yellow and blue atoms, respectively) and denoted as "screening" and b) removing three layer of atoms and denoted as "blunting". For both (a) and (b), the crack geometry is shown (i) at zero load, (ii) at the critical load for dislocation emission, and (iii) after dislocation emission. The triangles are the basic structural units through which the shear displacement along the plane of emission are analysed, as indicated. 
This is a post-print of the following article: Andric, Predrag; Curtin, W. A. Journal Of The Mechanics And Physics Of Solids $2017,23$. 315-337.. The formal publication is available at http://dx.doi.org/10.1016/j.jmps.2017.06.006 @ 2017. This manuscript version is made available under the CC-BY-NC-ND 4.0 license http://creativecommons.org/licenses/by-nc-nd/4.0/
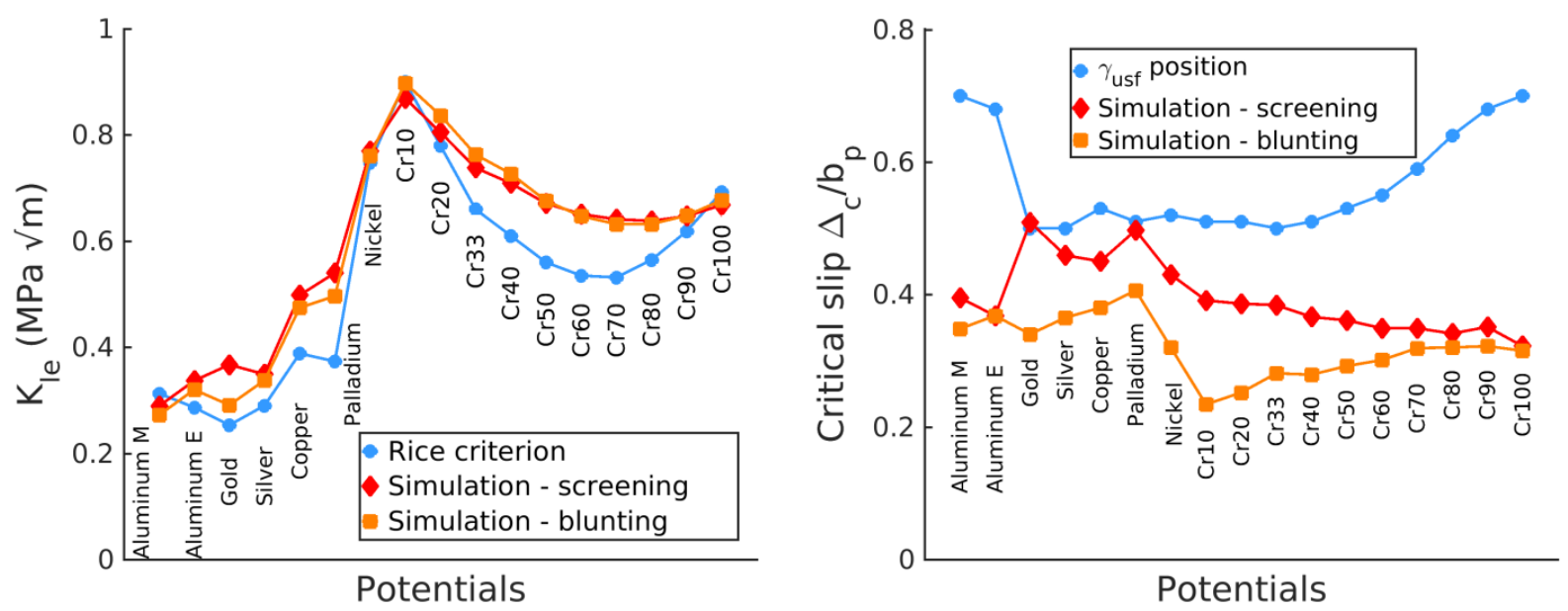

Figure 5: Results of simulations in Mode I for both "screening" and "blunting" models: a) Critical stress intensity factor $K_{I e}$ for crack-tip dislocation emission; b) Relative shear displacement of the structural units at the crack tip. In both (a) and (b), light blue symbols show the predictions of the Rice theory.

\begin{tabular}{c|c|ccc|ccccc}
\hline & & \multicolumn{3}{|c|}{ Screening } & \multicolumn{5}{c}{ Blunting } \\
Material & $\Delta_{\text {usf }} / \mathrm{b}_{\mathrm{p}}$ & $\mathrm{K}_{\text {sim }} / \mathrm{K}_{\text {Rice }}$ & $\Delta_{1} / \mathrm{b}_{\mathrm{p}}$ & $\Delta_{2} / \mathrm{b}_{\mathrm{p}}$ & $\mathrm{K}_{\text {sim }} / \mathrm{K}_{\text {Rice }}$ & $\Delta_{1} / \mathrm{b}_{\mathrm{p}}$ & $\Delta_{2} / \mathrm{b}_{\mathrm{p}}$ & $\Delta_{1}^{\prime} / \mathrm{b}_{\mathrm{p}}$ & $\Delta_{2}^{\prime} / \mathrm{b}_{\mathrm{p}}$ \\
\hline Al Mishin & 0.70 & 0.92 & 0.395 & 0.279 & 0.87 & 0.348 & 0.241 & 0.461 & 0.323 \\
Al Ercolessi & 0.68 & 1.18 & 0.368 & 0.258 & 1.12 & 0.368 & 0.280 & 0.484 & 0.356 \\
$\mathrm{Au}$ & 0.50 & 1.45 & 0.509 & 0.366 & 1.07 & 0.340 & 0.248 & 0.398 & 0.291 \\
$\mathrm{Ag}$ & 0.50 & 1.2 & 0.459 & 0.334 & 1.07 & 0.365 & 0.268 & 0.447 & 0.334 \\
$\mathrm{Cu}$ & 0.53 & 1.28 & 0.450 & 0.330 & 1.15 & 0.380 & 0.287 & 0.497 & 0.349 \\
$\mathrm{Pd}$ & 0.51 & 1.45 & 0.497 & 0.365 & 1.19 & 0.406 & 0.311 & 0.483 & 0.366 \\
$\mathrm{Ni}$ & 0.52 & 1.03 & 0.430 & 0.302 & 1.03 & 0.318 & 0.226 & 0.385 & 0.272 \\
$\mathrm{Cr} 10$ & 0.51 & 0.97 & 0.392 & 0.267 & 1.00 & 0.234 & 0.165 & 0.335 & 0.234 \\
$\mathrm{Cr} 20$ & 0.51 & 1.03 & 0.386 & 0.269 & 1.07 & 0.252 & 0.182 & 0.353 & 0.249 \\
$\mathrm{Cr} 33$ & 0.50 & 1.12 & 0.384 & 0.277 & 1.16 & 0.281 & 0.209 & 0.380 & 0.273 \\
$\mathrm{Cr} 40$ & 0.51 & 1.16 & 0.366 & 0.266 & 1.19 & 0.279 & 0.211 & 0.375 & 0.271 \\
$\mathrm{Cr} 50$ & 0.53 & 1.2 & 0.361 & 0.266 & 1.21 & 0.299 & 0.232 & 0.393 & 0.288 \\
$\mathrm{Cr} 60$ & 0.55 & 1.22 & 0.349 & 0.259 & 1.21 & 0.301 & 0.237 & 0.395 & 0.291 \\
$\mathrm{Cr} 70$ & 0.59 & 1.21 & 0.349 & 0.261 & 1.19 & 0.319 & 0.255 & 0.406 & 0.301 \\
$\mathrm{Cr} 80$ & 0.64 & 1.13 & 0.341 & 0.257 & 1.12 & 0.320 & 0.256 & 0.410 & 0.302 \\
$\mathrm{Cr} 90$ & 0.68 & 1.05 & 0.351 & 0.267 & 1.05 & 0.326 & 0.258 & 0.417 & 0.307 \\
$\mathrm{Cr} 100$ & 0.70 & 0.96 & 0.322 & 0.243 & 0.98 & 0.315 & 0.249 & 0.409 & 0.296 \\
\hline
\end{tabular}

Table 1: Positions of the $\gamma_{\mathrm{usf}}$, along with the simulation results in Mode I for screening and blunting given as the ratios of simulation $\mathrm{K}_{\mathrm{Ie}}$ and Rice prediction for $\mathrm{K}_{\mathrm{Ie}}$, and measured shear displacements of the first and second structural units at the point of emission (see Figure 4).

More importantly, Figure $5 \mathrm{~b}$ and Table 1 show the simulated and predicted results for 245 the critical crack tip shear displacement under Mode I loading. Here, we measure the shear deformation of the crack tip unit as $\Delta_{1}$ (see the initial crack in Figure 4). Due to very high normal stresses in the slip direction in Mode I, there can be some difference between $\Delta_{1}$ and $\Delta_{1}^{\prime}$ for some materials; physical aspects of this difference are explained in Appendix B. In Mode I loading, the difference between the Rice theory and simulation is notable: the 
This is a post-print of the following article: Andric, Predrag; Curtin, W. A. Journal Of The Mechanics And Physics Of Solids $2017,, 23$. 315-337.. The formal publication is available at http://dx.doi.org/10.1016/j.jmps.2017.06.006 ( ) 2017. This manuscript version is made available under the CC-BY-NC-ND 4.0 license http://creativecommons.org/licenses/by-nc-nd/4.0/

250 simulated instability point is often far below the value postulated by Rice, with differences of typically up to $40 \%$. From an energetic point of view, the energy release rate at displacements below $\sim b_{p} / 2$ is simply far too low to provide the unstable stacking fault energy necessary to nucleate a dislocation according to the Rice mechanism. Examining the results in Figure 5 further, we see that the screening case shows a critical slip at emission that is closer to the Rice prediction, but these cases also have a critical $K_{I e}$ that is much larger than the Rice prediction. For instance, in $\mathrm{Au}, \mathrm{Pd}$, and $\mathrm{Ag}$, the critical slip at the crack tip in the "screening" case is almost equal to the slip at the unstable stacking fault energy (the Rice prediction), but the $K_{I e}$ is $\sim 50 \%$ higher than the Rice prediction. Since the energy and the crack tip displacement are intimately coupled in the Rice theory, there is a fundamental problem with the Rice theory for Mode I loading. As we show in the next section, the problem lies in the fact that the Rice theory does not account for the creation of the surface step that accompanies dislocation emission in Mode I.

\section{Energy due to surface step creation during dislocation emission}

When a dislocation nucleates under Mode I loading, a surface step is created, as indicated in Figure 4. Figures 4a(ii),b(ii) are at the point of nucleation and, while the final state (Figures $4 \mathrm{a}(\mathrm{iii}), \mathrm{b}(\mathrm{iii})$ ) clearly shows the creation of a surface step, there is a nascent surface step and an associated partial step energy at the point of emission. The energy to create the emerging surface step is an additional energy cost for dislocation emission.

270 However, as seen previously in Figure 5b, the critical displacement at the point of dislocation emission is usually well below the Rice prediction. The energy of the inelastic slip along the stacking fault is therefore much lower than $\gamma_{u s f}$. The total energy at the critical displacement in Mode I is thus some fraction of $\gamma_{u s f}$ plus some fraction of the step energy. The critical displacement at the emission point is also not at the instability point predicted by the Rice theory and so a simple incorporation of a step energy into the Rice model, as done in early attempts to include the step [10-13], is not accurate. The dislocation nucleation differs significantly from the mechanism envisioned in the Rice theory, requiring an entirely new theory.

The first step toward a new theory involves investigation of the energy versus slip 280 displacement as the actual nucleation process takes place, including the energy associated with the emerging surface. We proceed in direct analogy to the usual computation of the GSF curve, using a method similar to that presented by Zamora et al. [33]. First, we create a non- 
This is a post-print of the following article: Andric, Predrag; Curtin, W. A. Journal Of The Mechanics And Physics Of Solids $2017,23$. 315-337.. The formal publication is available at http://dx.doi.org/10.1016/j.jmps.2017.06.006 ( ) 2017. This manuscript version is made available under the CC-BY-NC-ND 4.0 license http://creativecommons.org/licenses/by-nc-nd/4.0/

orthogonal simulation cell, oriented with $X=\left[\begin{array}{lll}\overline{1} & 1 & 2\end{array}\right], Y=\left[\begin{array}{lll}1 & 1 & 1\end{array}\right], Z=\left[\begin{array}{lll}\overline{1} & 1 & 0\end{array}\right]$. The simulation cell size is $6 \sqrt{6} a_{0}$ x $8 \sqrt{3} a_{0}$ x $2 \sqrt{2} a_{0}$ A. We apply periodic boundary conditions in [ $\left.\begin{array}{lll}1 & 1 & 2\end{array}\right]$ direction, while in $\left[\begin{array}{lll}1 & 1 & \overline{1}\end{array}\right]$ we apply free boundary conditions (see Figure 6a, b). Two identical crystal parts are defined and depicted as blue and red atoms, respectively. The interface between the blue and red atoms represents the slip plane. The initial crack tip geometry (blunting or screening) is created by (i) increasing of the simulation box size in $\left[\begin{array}{lll}\overline{1} & \overline{1} & \overline{2}\end{array}\right]$ direction and (ii) deleting extra atoms, as depicted in Figure 6a, b. We then rigidly slide the left crystal domain (blue atoms) relatively to the right crystal domain (red atoms). As in a standard GSF computation, relaxation is permitted only in the direction normal to the slip plane. We then compute energy change over a domain which includes three atoms on either side of the slip plane plus the crack tip atoms. The domain size in the [llll 112$]$ direction is sufficiently long so that atoms far from the crack tip are essentially bulk atoms. Figure 6c shows the energy change of each atom after slip of one partial Burgers vector (the final state after emission); the energy changes of the atoms just at the crack tip dominate the overall energy change. Along the slip plane away from the crack tip atoms, the energy is intrinsic stacking fault $\gamma_{s s f}$ but this energy is not visible in Figure $6 \mathrm{c}$ because it is overwhelmed by the energy of the crack tip atoms. At any slip displacement $\Delta$, we measure the energy change local to the crack tip atoms by subtracting the energy associated with the bulk GSF over all atoms in the domain except those at the crack tip, as outlined by the green line in Figures 6a,b. The remaining energy is the total energy, due to both the stacking fault and the step, associated with the slip of the atoms that define the crack tip structural unit. We divide this energy by the atomic spacing $\sqrt{6} b_{p} / 4$ along the [112] direction to obtain the crack tip slip energy (per unit area), which we call the nucleation energy, defined as

$$
\Psi_{n u c}(\Delta)=\Psi_{\text {step }}(\Delta)+\Psi_{g s f}(\Delta),
$$

where $\Psi_{\text {step }}(\Delta)$ is the energy associated with step creation at the crack tip and $\Psi_{g s f}(\Delta)$ is the bulk GSF energy contribution in the crack tip unit. Note that the two contributions in Eq. 9 are not independently separable in the simulations but we write Eq. 9 to indicate that, in the 310 absence of any step, the nucleation energy should still include the GSF energy. Figure $7 \mathrm{~b}$ shows $\Psi_{n u c}(\Delta)$ along with the standard GSF curve for the case of fcc Ni. Figure 7 exhibits several crucial features. First and foremost, unlike the GSF curve, there is no maximum in $\Psi_{n u c}(\Delta)$ at any slip $\Delta<b_{p}$. The absence of a maximum immediately precludes application of the Rice theory, which is based on a maximum energy $\left(\gamma_{u s f}\right)$ at which point the material offers no resistance to further slip. Second, the total crack tip energy is significantly larger 
This is a post-print of the following article: Andric, Predrag; Curtin, W. A. Journal Of The Mechanics And Physics Of Solids $2017,23$. 315-337.. The formal publication is available at http://dx.doi.org/10.1016/j.jmps.2017.06.006 @ 2017. This manuscript version is made available under the CC-BY-NC-ND 4.0 license http://creativecommons.org/licenses/by-nc-nd/4.0/

than the GSF energy. This is not surprising because the free surface energy is typically much larger than the unstable stacking fault energy. Third, the final value of the nucleation energy $\Psi_{n u c}\left(\Delta=b_{p}\right)$, which we call the surface step energy $\gamma_{\text {step }}$, is slightly lower than the flat surface energy $\gamma_{s}$ of the exposed crystalline facet. This difference is due to the local atomic structure of the crack tip. For all 17 potentials studied here, this ratio is $\gamma_{\text {step }} / \gamma_{s} \approx 0.7 \pm 0.05$. We can further verify that the surface step creation has no influence further along the slip plane away from the crack tip by analysing the energy changes of each triangular structural unit along the slip plane as a function of the relative slip $\Delta$ (see Figure $7 \mathrm{a})$. Figure $7 \mathrm{~b}$ shows that, even in unit 2 adjacent to the crack tip unit, the energy is nearly identical to the GSF energy. Therefore, the step energy is localized to the structural unit at the crack tip. This conclusion holds for every material studied here and will be used to develop a new theory for dislocation emission in the next section. The nucleation energy $\Psi_{n u c}(\Delta)$ and GSF energy $\Psi_{g s f}(\Delta)$ are computed for all materials studied here as shown in Figures 8a,b for both "screening" and "blunting" crack geometry; the conceptual points observed for Ni are valid in all cases. The small differences between "screening" and "blunting", not visible in Figures $8 \mathrm{a}, \mathrm{b}$, give rise to differences in the simulated $K_{I e}$, as we will see below.

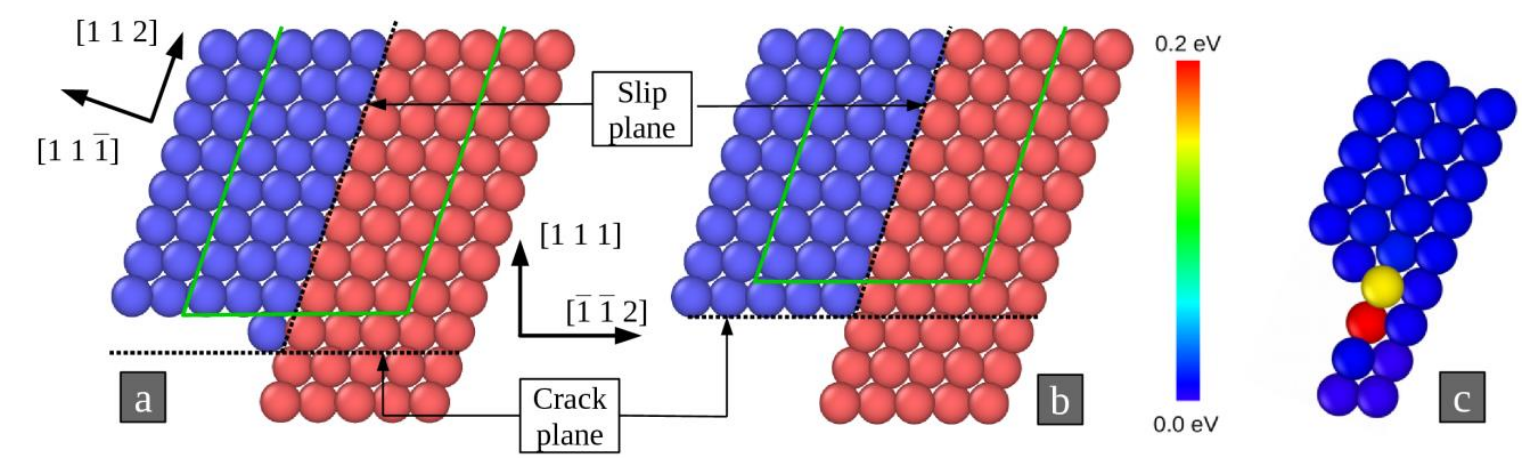

Figure 6: Computation of the nucleation energy a) for "blunting" crack geometry; b) for "screening" crack geometry; and c) energy change per atom in the blunting case after full slip (one partial Burgers vector) for Nickel. 
This is a post-print of the following article: Andric, Predrag; Curtin, W. A. Journal Of The Mechanics And Physics Of Solids $2017,23$. 315-337.. The formal publication is available at http://dx.doi.org/10.1016/j.jmps.2017.06.006 @ 2017. This manuscript version is made available under the CC-BY-NC-ND 4.0 license http://creativecommons.org/licenses/by-nc-nd/4.0/
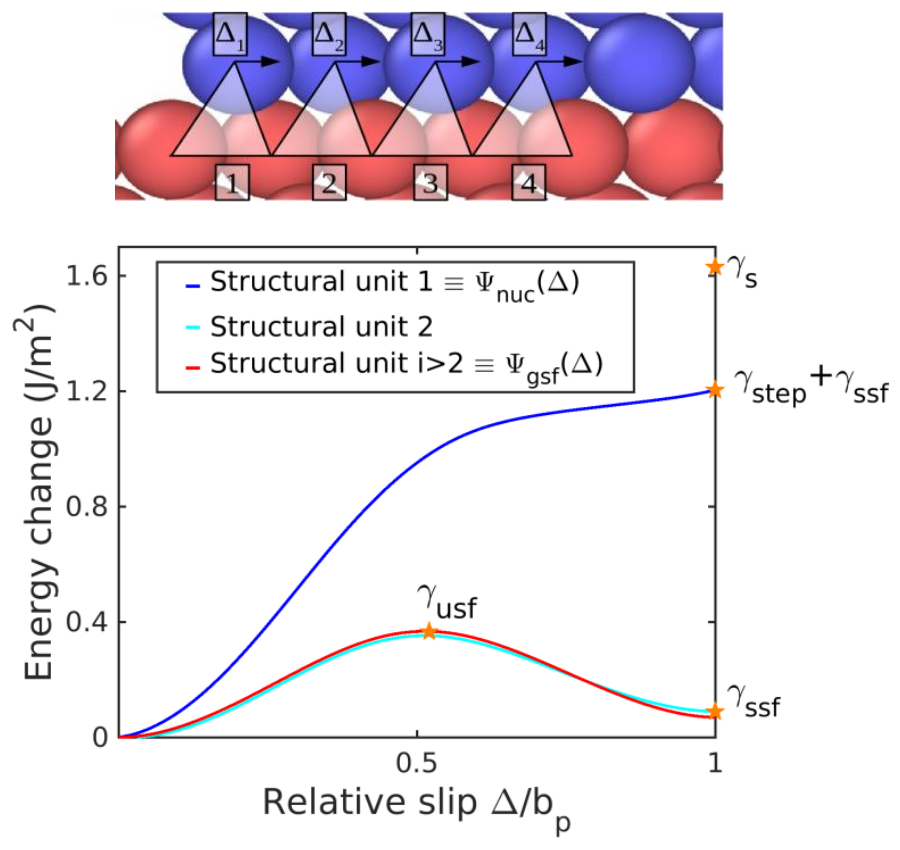

Figure 7: Slip energy change versus slip displacement, for successive atomic structural units along the slip plane computed for fcc Ni [19]; only the energy for the structural unit at the crack tip deviates significantly from the bulk GSF energy.
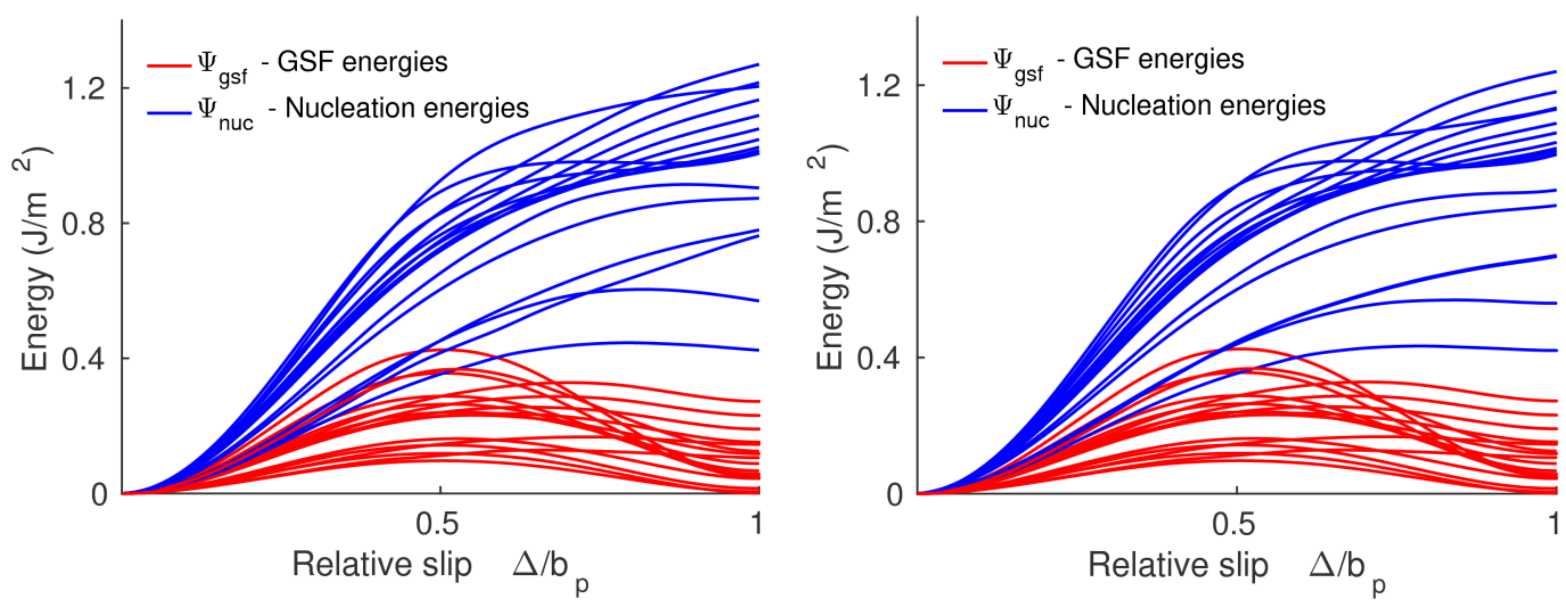

Figure 8: GSF energy (red lines) and nucleation energy (blue lines) versus relative slip for all fcc materials studied here: a) screening; b) blunting. GSF energies are identical in both figures.

\section{New theory for crack tip dislocation emission}

Since the nucleation energy $\Psi_{n u c}(\Delta)$ does not have a maximum, we deduce that emission must correspond to a local mechanical instability at the crack tip. Due to the high energy cost of shearing associated with step formation, the dislocation is "trapped" at the crack tip in a manner reminiscent of "lattice trapping" of a cleavage crack [18]. In "lattice trapping" for the cleavage crack problem, the precise force-displacement behavior of the crack-tip bond can restrain crack opening until the crack tip bond is mechanically unstable. 
This is a post-print of the following article: Andric, Predrag; Curtin, W. A. Journal Of The Mechanics And Physics Of Solids $2017,23$. 315-337.. The formal publication is available at http://dx.doi.org/10.1016/j.jmps.2017.06.006 ( ) 2017. This manuscript version is made available under the CC-BY-NC-ND 4.0 license http://creativecommons.org/licenses/by-nc-nd/4.0/

At this point, the applied load $K_{I}$ exceeds the thermodynamic Griffith value $K_{I c}$. In the dislocation emission problem here, the shear stress needed to shear the crack tip unit up to the unstable point of dislocation emission is higher than that corresponding to the GSF energy. Once the shear of the crack tip unit reaches a critical level at which the crack tip unit becomes unstable, the dislocation then move away unstably along the slip plane. The weaker restoring stresses further along the slip plane, due only to the GSF energy, are unable to impede the dislocation nucleation.

While the new theory will be fundamentally different from the Rice theory, we retain key assumptions consistent with the Rice analysis (see Figure 9). Most importantly, we assume that (i) only the shear resistance along the slip plane controls the nucleation process, (ii) all non-linear behavior is confined to the slip plane, and (iii) that the shear displacement distribution $\Delta_{1}, \Delta_{2}, \ldots$ along the slip plane is the same in Modes I and II. This last assumption has been explicitly verified as shown in Appendix C, where we demonstrate that, for a specified displacement $\Delta_{1}$ at the crack tip, the remaining displacements $\Delta_{2}, \Delta_{3}, \ldots$ along the slip plane are essentially the same in Mode I and Mode II loadings. The main difference between the new theory and the Rice theory will be in the crack tip constitutive behavior, i.e. the resistance of the crack tip structural unit to shearing due to the emergence of the step in Mode I but not in Mode II, as shown already in Figure 7b. The other difference with Rice analysis will be that we deal only with the total shear displacements $\Delta$; we find no need to introduce the inelastic slip measure $\delta$ and in this aspect we are consistent with the analysis of Schoeck [14].

We start by analyzing the case with zero step energy; this analysis thus also applies to Mode II and will reveal the Rice solution from a different perspective. For zero step energy, the energy versus slip is only the GSF energy. The corresponding GSF "restoring" shear stress $\tau_{\text {res }}$ across the slip plane is the derivative of the energy. We use a sinusoidal Peierls model so that $\tau_{\text {res }}=\tau_{g s f}=\left(\mu b_{p} / 2 \pi h\right) \sin \left(2 \pi \Delta / b_{p}\right)$, as shown in Figure 10a. We now focus on the crack tip structural unit and assume that all non-linear behavior is confined to this crack tip structural unit, i.e. shear deformations further along the slip plane remain in the (nearly) linear domain of the Peierls curve. Then, a remote applied $K_{I}$ generates a shear stress $\tau_{a p p, 0}$ on the crack-tip unit that is linear in $K_{I}$. As the shear displacement $\Delta$ of the crack tip unit increases, the applied shear decreases linearly as $\tau_{a p p}=\tau_{a p p, 0}-\mu \Delta / h(\beta-1)$, as indicated in Figure 10a. Here, the constant $\beta$ is the crack-tip Green's function for shear in the lattice; $\beta=2$ for an isotropic continuum and varies between 1.4 and 2.3 for the anisotropic fcc materials studied here (see Appendix D). 
This is a post-print of the following article: Andric, Predrag; Curtin, W. A. Journal Of The Mechanics And Physics Of Solids $2017,23$. 315-337.. The formal publication is available at http://dx.doi.org/10.1016/j.jmps.2017.06.006 ( ) 2017. This manuscript version is made available under the CC-BY-NC-ND 4.0 license http://creativecommons.org/licenses/by-nc-nd/4.0/

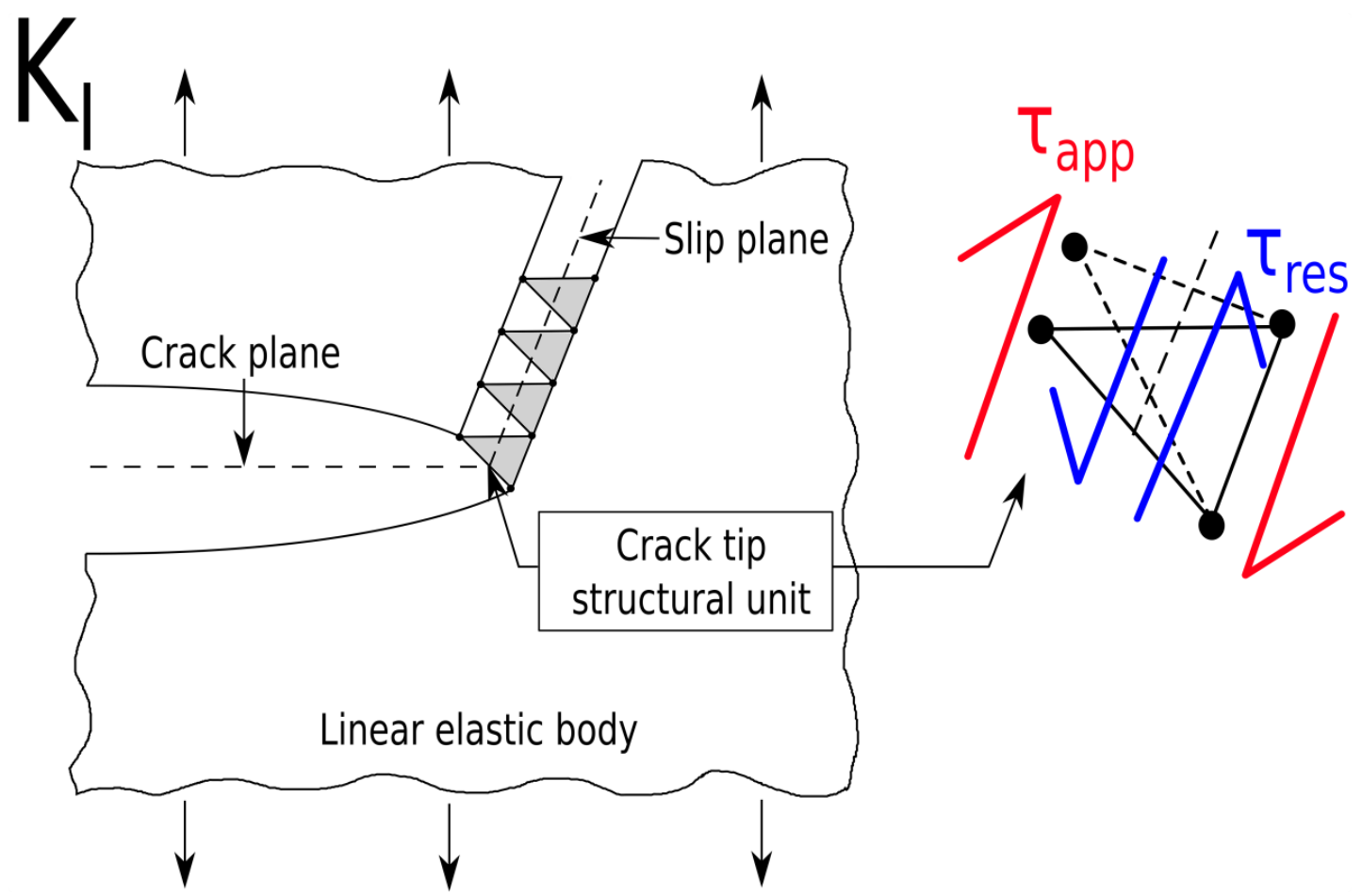

Figure 9: a) Linear elastic body with semi-infinite crack under pure Mode I loading surrounding the dislocation emission plane that is characterized by atomic structural units along the slip plane; b) Local stresses acting on the crack tip structural unit: an applied stress due to the remote applied $\mathrm{K}$ load and a restoring stress due to the shear resistance of the crack tip structural unit, which together determine the local equilibrium shear deformation (dashed lines).
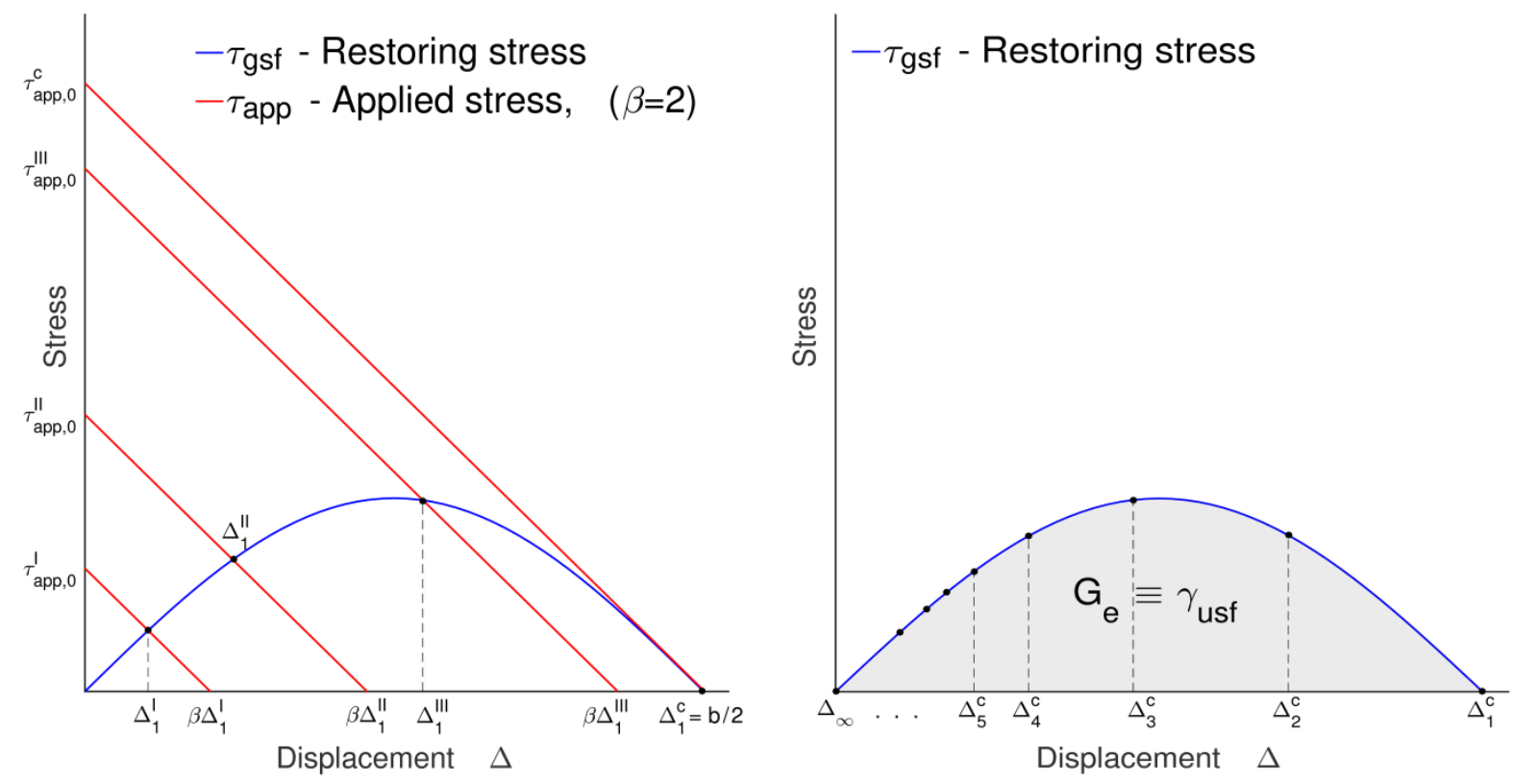

Figure 10: Graphical construction for the equilibrium shear displacement $\Delta_{1}$ of the crack tip unit in Mode II loading or in Mode I loading when no step is present, for several different far-field loadings. Solid blue line: crack tip restoring stress versus crack tip shear displacement due to GSF energy only; Red lines: crack tip applied shearing stress versus shear displacement. b) Critical shear displacements $\Delta_{1}$, $\Delta_{2}, \ldots$ along the slip plane at the point of dislocation emission, with critical energy release rate corresponding to the shaded area. 
This is a post-print of the following article: Andric, Predrag; Curtin, W. A. Journal Of The Mechanics And Physics Of Solids $2017,23$. 315-337.. The formal publication is available at http://dx.doi.org/10.1016/j.jmps.2017.06.006 @ 2017. This manuscript version is made available under the CC-BY-NC-ND 4.0 license http://creativecommons.org/licenses/by-nc-nd/4.0/

At any given applied $K_{I}$, and thus any given $\tau_{a p p, 0}$, the equilibrium crack tip displacement $\Delta_{1}$ is given by the condition $\tau_{a p p}=\tau_{\text {res }}$, as shown graphically in Figure 10a for several values of $\tau_{a p p, 0}$ with $\beta=2$. For the sequence of applied stresses $\left(\tau_{a p p, 0}^{\prime}\right.$, $\left.\tau_{a p p, 0}^{\prime \prime} \ldots\right)$, the corresponding equilibrium shear displacements $\left(\Delta_{1}^{\prime}, \Delta_{1}^{\prime \prime}, \ldots\right)$ are stable equilibrium points because $d \tau_{a p p} / d \Delta<d \tau_{\text {res }} / d \Delta$ for all $0<\Delta<b_{p} / 2$. There is no mechanical instability until $\Delta_{1}=\Delta_{1}^{c}=b_{p} / 2$. The stress $\tau_{a p p, 0}^{c}$ at this instability is then proportional to $K_{I e}$. The assumption of linearity for all units ahead of the crack tip unit is not really true: in the more general case, the crack tip units deform stably along the GSF curve until the crack tip unit becomes unstable. The instability could then occur prior to $\Delta_{1}=b_{p} / 2$; in other words, some lattice trapping can occur in Mode II even without the step, and this can account for the deviation in predicting $\mathrm{K}_{\text {IIe }}$ in the $\mathrm{Cr} 60-\mathrm{Cr} 100$ potentials. However, the spatial range of the GSF stress versus displacement corresponds, in the language of the "lattice trapping" cleavage problem [18], to a relative long-range force law with very small lattice trapping. The instability thus usually occurs very near, or at, $\Delta_{1}=\Delta_{1}^{c}=b_{p} / 2$, with $\Delta_{2}^{c}, \Delta_{3}^{c} \ldots$ as shown in Figure $10 \mathrm{~b}$, and hence trapping is generally small in Mode II. At the instability point, all atoms move forward to the next stable position, such that $\Delta_{2}^{c} \rightarrow \Delta_{1}^{c}, \Delta_{3}^{c} \rightarrow \Delta_{2}^{c}, \ldots$ The total energy required to reach the instability point is then equal to the energy required to take the crack tip unit from $\Delta_{1}=0$ to $\Delta_{1}=\Delta_{1}^{c}=b_{p} / 2$. This energy is equal to the area under the $\tau_{g s f}$ curve, which in turn is precisely $\gamma_{u s f}$, as indicated in Figure 10b. This construction is the discrete analog to the continuous cohesive zone model that is implicit in the Rice theory, but using the total shear displacement $\Delta$ and GSF energy $\Psi_{g s f}(\Delta)$ rather than the displacement discontinuity $\delta$ and energy $\Phi(\delta)$.

We now use the same general analysis to consider the case of dislocation emission when a surface step is created. Again, we assume that only the crack tip deforms non-linearly. Due to the step creation, the energy of the crack tip unit is $\Psi_{n u c}(\Delta)=\Psi_{\text {step }}(\Delta)+\Psi_{g s f}(\Delta)$ 410 and the restoring stress is $\tau_{\text {res }}=\mathrm{d} \Psi_{n u c}(\Delta) / d \Delta$. Figure 11 shows the restoring stress for a Peierls model of the nucleation energy, $\Psi_{n u c}(\Delta)$ and $\tau_{r e s}=g\left(\mu b_{p} / 2 \pi h\right) \sin \left(2 \pi \Delta / b_{p}\right)$, where $g=\tau_{r e s} / \tau_{g s f}$ is the factor by which the restoring stress with the step exceeds the restoring stress when there is no step creation. However, the surrounding material remains elastic. Therefore, the applied stress remains exactly the same as before, $\tau_{a p p}=\tau_{a p p, 0}-\mu \Delta / h(\beta-1)$. With increasing applied stress $\tau_{a p p, 0}$, the stable equilibrium shear displacement $\Delta_{1}$ of the crack tip unit evolves stably as shown in Figure 11. A mechanical instability, corresponding to dislocation nucleation, then occurs at the applied 
This is a post-print of the following article: Andric, Predrag; Curtin, W. A. Journal Of The Mechanics And Physics Of Solids $2017,23$. 315-337.. The formal publication is available at http://dx.doi.org/10.1016/j.jmps.2017.06.006 @ 2017. This manuscript version is made available under the CC-BY-NC-ND 4.0 license http://creativecommons.org/licenses/by-nc-nd/4.0/

stress $\tau_{a p p, 0}^{c, s t e p}$ where the equilibrium shear displacement becomes metastable, i.e. when $\tau_{a p p}=$ $\tau_{\text {res }}$ and $d \tau_{a p p} / d \Delta=d \tau_{\text {res }} / d \Delta$, as indicated in Figure 11 . When $g>1.5$, and for $\beta=2$, the instability occurs at $\Delta_{1}^{c} \ll b_{p} / 2$ and the shear displacements $\Delta_{2}^{c}, \Delta_{3}^{c} \ldots$ ahead of the crack tip remain (nearly) in the linear range of the GSF stress curve, as indicated in Figure 12a. The ratio of the remote applied stress intensity at the instability point for the step case (Mode I) to that of the no-step case (the Rice model) is equal to the ratio of the critical stresses,

$$
\tau_{a p p, 0}^{c, \text { step }} / \tau_{a p p, 0}^{c}=K_{\text {Ie,step }} / K_{\text {Ie,Rice }}
$$

As seen graphically in Figure 11, the fractional increase in the remote stress intensity factor is larger than, but not significantly larger than, the Rice value. The "trapping" of the nucleating dislocation at the crack tip due to the extra energy of the step thus leads to a critical shear displacement instability that is lower than the Rice value but at a $K_{I e}$ that is larger than the Rice value.

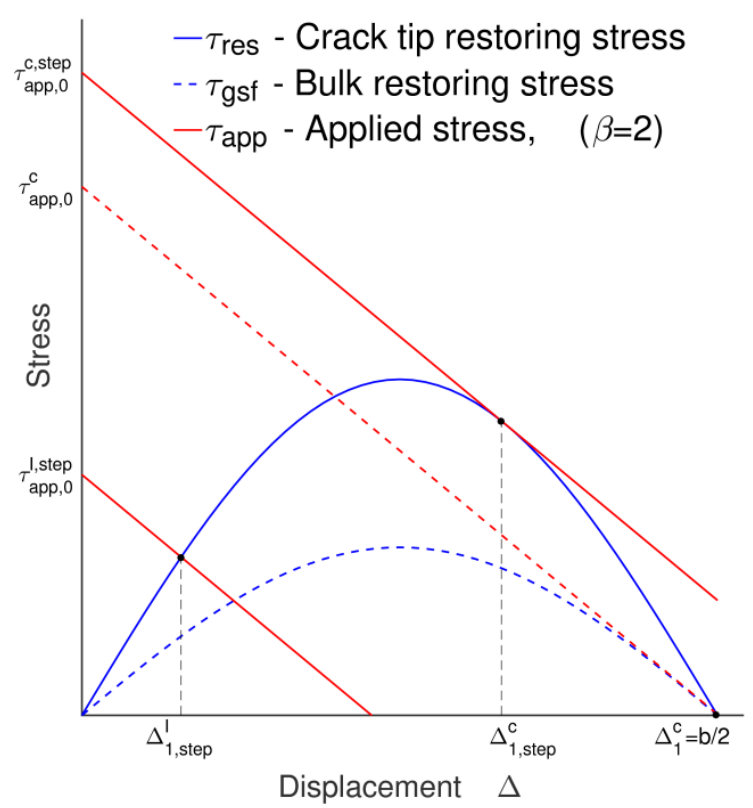

Figure 11: Graphical construction for the equilibrium shear displacement $\Delta_{1}$ of the crack tip unit when a step is created during emission in Mode I loading and all other displacements $\Delta_{2}, \Delta_{3}, \ldots$ remain in the linear regime, for several different far-field loadings. Solid blue line: crack tip restoring stress versus shear displacement including the step energy; Red lines: crack tip applied shearing stress versus shear displacement; for reference, dashed blue line shows the crack tip restoring stress versus shear displacement due only to the stacking fault energy.

The graphical analysis using the Peierls representation, as shown in Figure 11, can be executed numerically for any desired values of the two relevant material parameters $\beta$ and $g$. Table 2 shows computed results for a range of typical values in real materials. At fixed $\beta$, increasing the step energy (increasing $g$ ) leads to increasing critical stress intensity for emission and decreasing critical crack tip shear displacement. These results are broadly consistent with the Mode I simulation results. Three important aspects merit comment. First, 
This is a post-print of the following article: Andric, Predrag; Curtin, W. A. Journal Of The Mechanics And Physics Of Solids $2017,23$. 315-337.. The formal publication is available at http://dx.doi.org/10.1016/j.jmps.2017.06.006 @ 2017. This manuscript version is made available under the CC-BY-NC-ND 4.0 license http://creativecommons.org/licenses/by-nc-nd/4.0/

the theory predicts a continuous transition from emission controlled by $\gamma_{u s f}$ (Rice theory) to emission controlled by the step energy. Second, as seen for the case of $g=1.5$, the increase in $K_{I e}$ above the Rice value is quite small even when the step energy is an appreciable fraction of $\gamma_{u s f}$. Third, the analysis is independent of the slip plane inclination angle $\theta$ because the same nominal step is created at any $\theta \neq 0^{0}$ and rotation of the stress field accounts for all differences in the "applied" stresses (see Appendix E).

\begin{tabular}{|c|c|c|c|c|c|c|}
\hline \multirow[b]{2}{*}{$g$} & \multicolumn{2}{|l|}{$\beta=1.5$} & \multicolumn{2}{|l|}{$\beta=1.75$} & \multicolumn{2}{|l|}{$\boldsymbol{\beta}=\mathbf{2}$} \\
\hline & $K_{\text {Ie,step }} / K_{\text {Ie,Rice }}$ & $\Delta_{c}$ & $\mathbf{K}_{\text {Ie,step }} / \mathbf{K}_{\text {Ie,Rice }}$ & $\Delta_{c}$ & $\mathbf{K}_{\text {Ie,step }} / \mathbf{K}_{\text {Ie,Rice }}$ & $\Delta_{c}$ \\
\hline 1.5 & 1 & 0.5 & 1.01 & 0.42 & 1.09 & 0.36 \\
\hline 2 & 1 & 0.5 & 1.09 & 0.36 & 1.22 & 0.33 \\
\hline 2.5 & 1.03 & 0.4 & 1.18 & 0.33 & 1.36 & 0.31 \\
\hline 3 & 1.09 & 0.36 & 1.29 & 0.32 & 1.5 & 0.3 \\
\hline
\end{tabular}

Table 2: Ratio of critical stress intensity factors with $\left(\mathrm{K}_{\mathrm{Ie}, \mathrm{step}}\right)$ and without $\left(\mathrm{K}_{\mathrm{Ie}, \mathrm{Rice}}\right)$ the surface step, for various values of the $g=\tau_{\text {res }} / \tau_{g s f}$ and crack tip Green's function parameter $\beta$, as computed using the Peierls model of Figure 11.

In realistic cases, the slip displacements away from the crack tip can again become (slightly) non-linear. In this case, the system softens and the simple graphical analysis based on non-linearity only at the crack tip is insufficient. However, at the instability point, the shear displacements again simply shift as $\Delta_{2}^{c} \rightarrow \Delta_{1}^{c}, \Delta_{3}^{c} \rightarrow \Delta_{2}^{c}$, etc. So, even when non-linearity extends beyond the crack tip structural unit, the energy that must be provided by the applied field to reach the point of instability is computed as the area under the curve shown in Figure 12a. The critical energy for emission can thus be computed in terms of the critical displacements $\Delta_{1}^{c}, \Delta_{2}^{c}$. There are two contributions, one from the crack tip unit that follows $\Psi_{n u c}$ and another from all other units that follow the $\Psi_{g s f}$ energy function, so that

$$
J=\int_{\Delta_{2}^{c}}^{\Delta_{1}^{c}} \tau_{r e s} d \Delta+\int_{0}^{\Delta_{2}^{c}} \tau_{g s f} d \Delta,
$$

which yields

$$
J=\Psi_{n u c}\left(\Delta_{1}^{c}\right)-\Psi_{n u c}\left(\Delta_{2}^{c}\right)+\Psi_{g s f}\left(\Delta_{2}^{c}\right) \equiv G_{I e}
$$

as shown graphically in Figure 12. The critical stress intensity factor is then computed using the standard relationship between $K$ and $G$,

$$
K_{I e}=\sqrt{G_{I e} O(\theta, \phi)} / F_{12}(\theta)
$$


This is a post-print of the following article: Andric, Predrag; Curtin, W. A. Journal Of The Mechanics And Physics Of Solids $2017,23$. 315-337.. The formal publication is available at http://dx.doi.org/10.1016/j.jmps.2017.06.006 @ 2017. This manuscript version is made available under the CC-BY-NC-ND 4.0 license http://creativecommons.org/licenses/by-nc-nd/4.0/

The analysis thus resembles the Rice theory, but emission is controlled by reaching a critical crack tip displacement associated with a mechanical instability due to the step formation.

Furthermore, if the maximum crack tip restoring stress is skewed toward higher displacements (see Figure 12b), which arises in some of the atomistic systems (derivatives of curves shown in Figure 8), then the instability can be shifted toward larger displacements, even reaching $\Delta_{1}^{c} \sim b_{p} / 2$, but then with a much greater energy and hence a much greater $K_{I e}$. Such a situation prevails in materials such as Au and Pd (see Figure 5). So, a measurement of emission at $\sim b_{p} / 2$ does not at all imply that the Rice model is applicable.
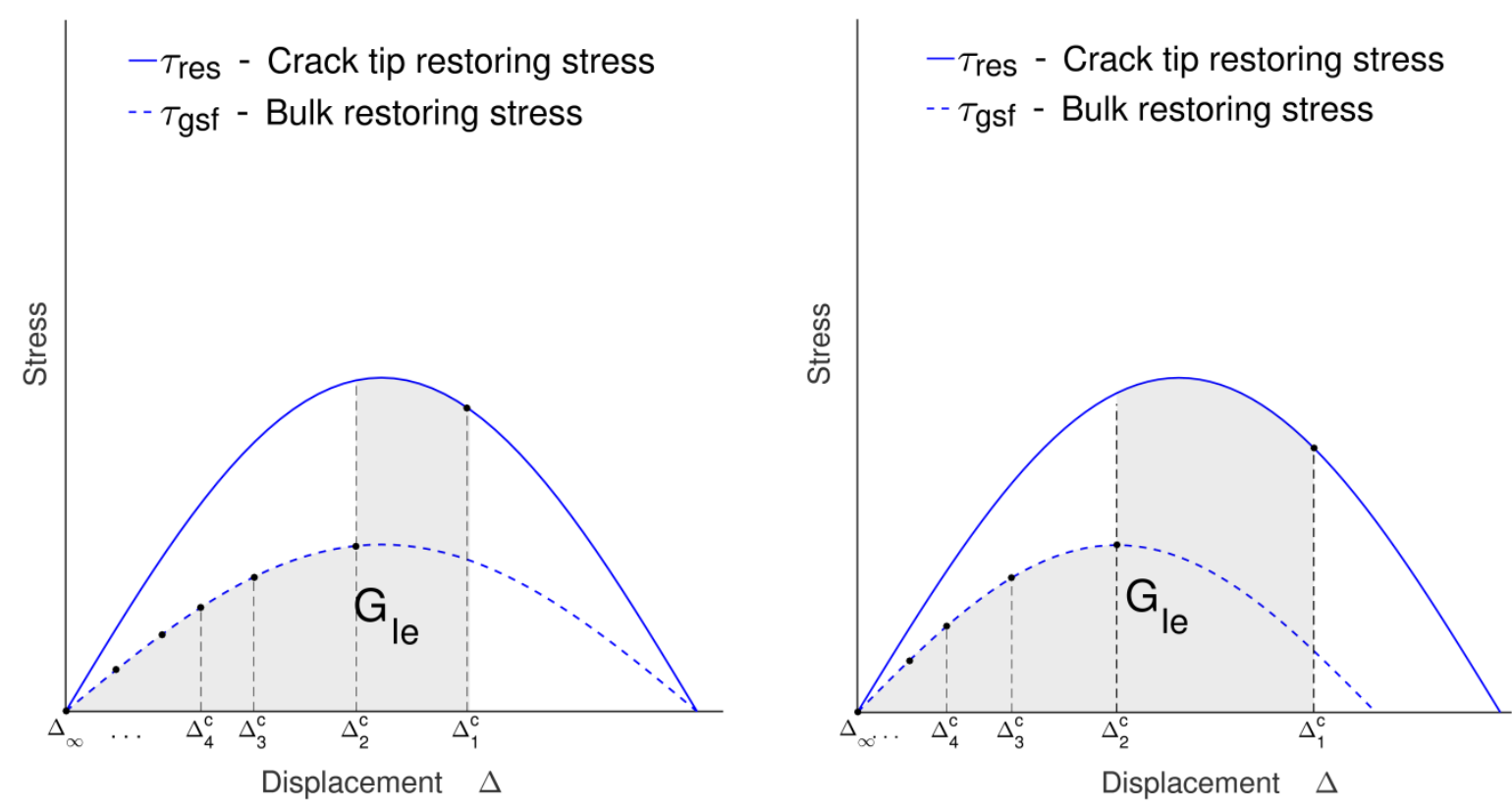

Figure 12: a) Critical shear displacements $\Delta_{1}, \Delta_{2}, \ldots$ along the slip plane at the point of dislocation emission, for the realistic case corresponding to some non-linearity in $\Delta_{2}, \Delta_{3}, \ldots$ beyond the cracktip structural unit. b) Critical shear displacements $\Delta_{1}, \Delta_{2}, \ldots$ along the slip plane at the point of dislocation emission, when the crack tip restoring stress is skewed toward higher displacement. In both figures the associated critical energy release rate corresponds to the shaded area.

\section{Validation of the new theory}

The previous section presented an analytical model that highlights the controlling physics/mechanics of the crack tip dislocation emission, and clearly rationalizes the origins of the deviations from the Rice theory. The analysis predicts the critical crack tip shear displacement $\Delta_{1}^{c}$ but assumes non-linearity to exist only in the crack tip unit. Even with some non-linearity away from the crack tip unit, the new model can predict the critical shear displacement $\Delta_{1}^{c}$ and the critical energy release rate $G_{I e}$ (as shown in Figure 12) for some 
This is a post-print of the following article: Andric, Predrag; Curtin, W. A. Journal Of The Mechanics And Physics Of Solids $2017,23$. 315-337.. The formal publication is available at http://dx.doi.org/10.1016/j.jmps.2017.06.006 @ 2017. This manuscript version is made available under the CC-BY-NC-ND 4.0 license http://creativecommons.org/licenses/by-nc-nd/4.0/

480

simulated materials ( $\mathrm{Ni}, \mathrm{Cu}$ and $\mathrm{Cr} 10-\mathrm{Cr} 40)$ in very good agreement with simulation results. However, in most of the simulated materials, the non-linear shear displacements further along the slip plane do not allow for direct application of the analytic theory. While non-linear behavior in the second structural unit could be included, such a complication is beyond the scope of this paper. Instead, we validate the new theory for $\mathrm{K}_{\mathrm{Ie}}$ by using the simulated values of the critical shear displacement (see Table 1) as input to compute $\mathrm{G}_{\mathrm{Ie}}$, as shown in Figure 12. While not a fully independent prediction of both $\Delta_{1}^{c}$ and $\mathrm{K}_{\mathrm{Ie}}$, this approach nonetheless quantitatively demonstrates key aspects of the theory.

First, we assess the accuracy of the new theory for cracks formed by "screening". Figure $13 \mathrm{a}$ shows the critical stress intensity factor $K_{I e}$ as predicted using the new theory (Eqs. 11-13); also shown are the simulation results and the Rice predictions. The predictions of the new theory are in excellent agreement with simulations, and generally better than, or comparable to, the Rice predictions. The new theory always predicts slightly higher results than found in the simulations, which likely reflects the limits of all models that use elasticity plus a non-linear slip model only along the slip plane. The overestimations found for $\mathrm{Cr} 10$ and $\mathrm{Cr} 20$ are cases with high normal stresses at the crack tip where opening softening may be more important (see below).

Next, we examine the accuracy of the new theory for cracks formed by blunting. Note that the Rice theory does not distinguish between these two cases, aside from approximate attempts to deal with elliptical crack tips [27]. Figure $13 \mathrm{~b}$ shows that the predicted $K_{I e}$ value is very close to the Rice value, with overall comparable agreement (sometimes slightly better, sometimes slightly worse) than the Rice prediction. Only Cr10 and Cr20 are notably off from the simulations. Recall, however, that the critical crack tip shear for blunting is much smaller than the Rice prediction, so that the physical model associated with the new theory is much more accurate overall than the Rice model. The present model thus also accounts for the differences in both $K_{I e}$ and $\Delta_{1}^{c}$ between "screening" and "blunting". As shown in Figure 14 for the specific cases of $\mathrm{Ni}$ and $\mathrm{Cu}$ (and also for both $\mathrm{Al}, \mathrm{Au}, \mathrm{Ag}, \mathrm{Pd}, \mathrm{Cr} 100$ and $\mathrm{Cr} 90$ potentials in Figure 8), the restoring stress for "blunting" is shifted to slightly lower shear displacements as compared to "screening", leading to smaller predicted $K_{I e}$ and $\Delta_{1}^{c}$. 
This is a post-print of the following article: Andric, Predrag; Curtin, W. A. Journal Of The Mechanics And Physics Of Solids $2017,23$. 315-337.. The formal publication is available at http://dx.doi.org/10.1016/j.jmps.2017.06.006 ( 2017 . This manuscript version is made available under the CC-BY-NC-ND 4.0 license http://creativecommons.org/licenses/by-nc-nd/4.0/
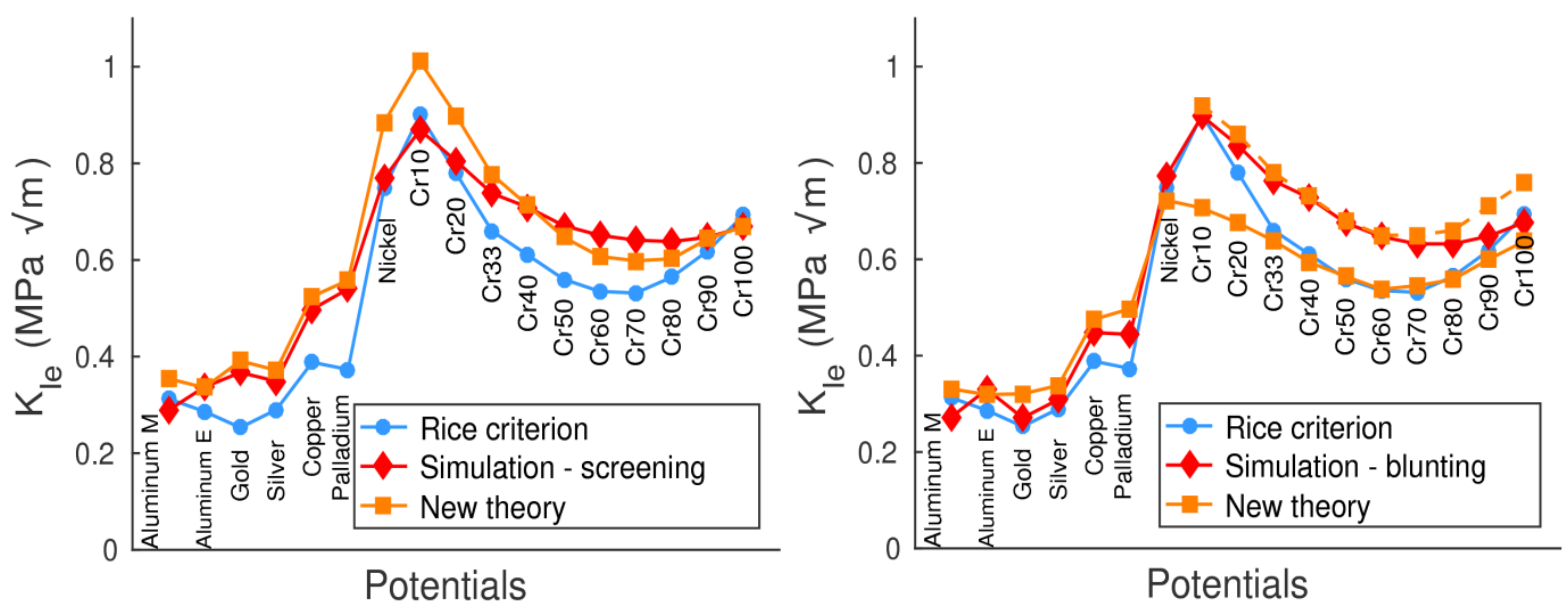

Figure 13: a) Critical stress intensity factor for dislocation emission (Theory: orange; Simulation: red; Rice: blue line) for a) cracks formed by "screening"; b) cracks formed by "blunting". Orange dashed line computed with $\Delta_{1}$ ' crack tip displacement.

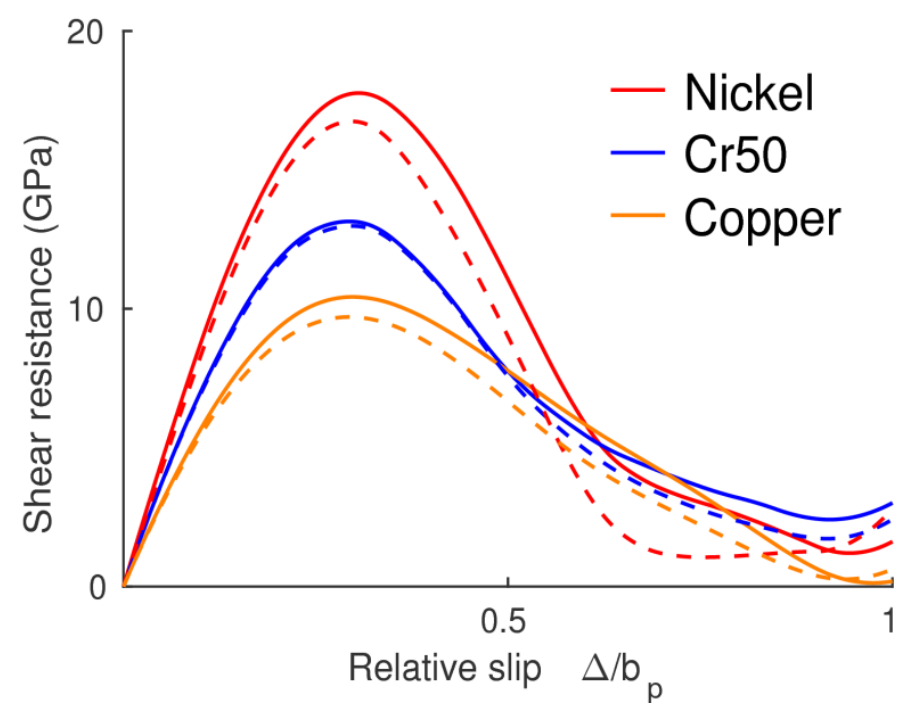

Figure 14: Crack tip restoring stress for crack formed by screening (solid lines) and for crack formed by blunting (dashed lines) in $\mathrm{Ni}, \mathrm{Cr} 50$ and $\mathrm{Cu}$.

The deviation of the new theory from the simulations for the $\mathrm{Cr} 10-\mathrm{Cr} 80$ potentials lies in the fact that the crack tip shearing energy is strongly affected by the normal stress parallel to the slip plane. The measured critical $\Delta_{1}^{c}$ for these potentials is actually below the displacement at the maximum restoring stress, and so there cannot be an instability based on $\Delta_{1}^{c}$ alone. In these cases, the normal stress acting at the crack tip stretches the crack tip unit (see Appendix B) so that $\Delta_{1}$ is not an accurate measure of the average crack tip shear. Instead, the shear displacement $\Delta_{1}^{\prime}$ better reflects the shearing of the crack tip unit. In addition, the restoring stress for these potentials (shown in Figure 14 for the specific case of $\mathrm{Cr} 50$ ) is not affected by the crack geometry and so crack geometry does not influence the simulated $K_{I e}$ (see Figure $5 \mathrm{a}$ ), and thus $\Delta_{1}$ in the screening case is very close to $\Delta_{1}^{\prime}$ in the blunting case (see 
This is a post-print of the following article: Andric, Predrag; Curtin, W. A. Journal Of The Mechanics And Physics Of Solids $2017,23$. 315-337.. The formal publication is available at http://dx.doi.org/10.1016/j.jmps.2017.06.006 ( ) 2017. This manuscript version is made available under the CC-BY-NC-ND 4.0 license http://creativecommons.org/licenses/by-nc-nd/4.0/

Table 1). As shown in Figure $13 \mathrm{~b}$ by the dashed line, predictions of the theory using the measured $\Delta_{1}^{\prime}$ and the same $\Psi_{\text {nuc }}(\Delta)$ are in very good agreement with the simulations. This difference shows that the precise deformation at the crack tip structural unit affects the critical stress intensity for emission by modest amounts, but these amounts can explain the differences between sharp "screened" cracks and "blunted" cracks, a feature absent in the Rice theory.

\section{Approximate model for prediction of the crack-tip dislocation emission}

The analysis in the previous section demonstrates the quantitative success of the conceptually new model. However, the predictions in Figures 13a,b use the simulation values for the critical shear displacement. Unlike the simple Peierls model (Figures 10, 11), the instability point $\Delta_{1}^{c}$ has not been predicted; the effects of non-linearities and the precise shear vs. displacement behaviour beyond the maximum shear resistance preclude analytic analysis. Furthermore, we seek an analytic model that does not require direct atomistic simulations of the crack problem since there is no need for a model if one only needs to execute a standard molecular statics crack simulation. Thus, we aim for simplified models that predict $K_{I e}$ in terms of only the easily-computable (i) GSF curve $\Psi_{\text {gsf }}$ and nucleation energy curve $\Psi_{\text {nuc }}$ or

535 (ii) unstable stacking fault energy $\gamma_{u s f}$ and surface energy $\gamma_{s}$.

For the simplified model which involves only $\Psi_{\mathrm{gsf}}$ and $\Psi_{\text {nuc }}$ it is necessary to determine the critical crack tip shear displacements $\Delta_{1}^{c}, \Delta_{2}^{c}$. The analytical model of Section $\mathrm{V}$, along with the results given in Table 2, shows that the critical value $\Delta_{1}^{c}$ is weakly dependent on the step energy once the step energy is somewhat larger than the GSF energy.

540 These results are also consistent with the simulations. Based on these observations we deduce that single value of $\Delta_{1}^{c}$ is sufficient for any material and can be used in the approximate model. Analyzing the critical crack tip shear displacements shown in Table 1, we can estimate $\Delta_{1}^{c} \approx 0.39 b_{p}$ for "screening" and $\Delta_{1}^{c} \approx 0.33 b_{p}$ for "blunting", which are the averages across the entire set of simulation results for "screening" and "blunting", respectively. For both crack configurations we find $\Delta_{2}^{c} / \Delta_{1}^{c} \approx 0.7$; this is not surprising because the ratio $\Delta_{2}^{c} / \Delta_{1}^{c}$ is determined mainly by elasticity and so is not strongly dependent on $\Delta_{1}^{c}$ nor crack geometry. Using these pairs of values for all materials, and $\Psi_{\text {nuc }}$ and $\Psi_{\text {gsf }}$ for each specific material, we compute $K_{I e}$ via Eqs. 12-13 and obtain the results shown in Figures 15a,b. This approximate solution is again in very good agreement with the simulations across the entire range of materials. This estimate works well even when the $\Delta_{1}^{c}$ deviates from the above estimated 
This is a post-print of the following article: Andric, Predrag; Curtin, W. A. Journal Of The Mechanics And Physics Of Solids $2017,23$. 315-337.. The formal publication is available at http://dx.doi.org/10.1016/j.jmps.2017.06.006 @ 2017. This manuscript version is made available under the CC-BY-NC-ND 4.0 license http://creativecommons.org/licenses/by-nc-nd/4.0/

value because (i) there is some cancellation of errors, (ii) $\Delta_{2}^{c} / \Delta_{1}^{c} \approx 0.7$ is retained, and (iii) $K_{I e}$ scales only with the square root of the critical energy.
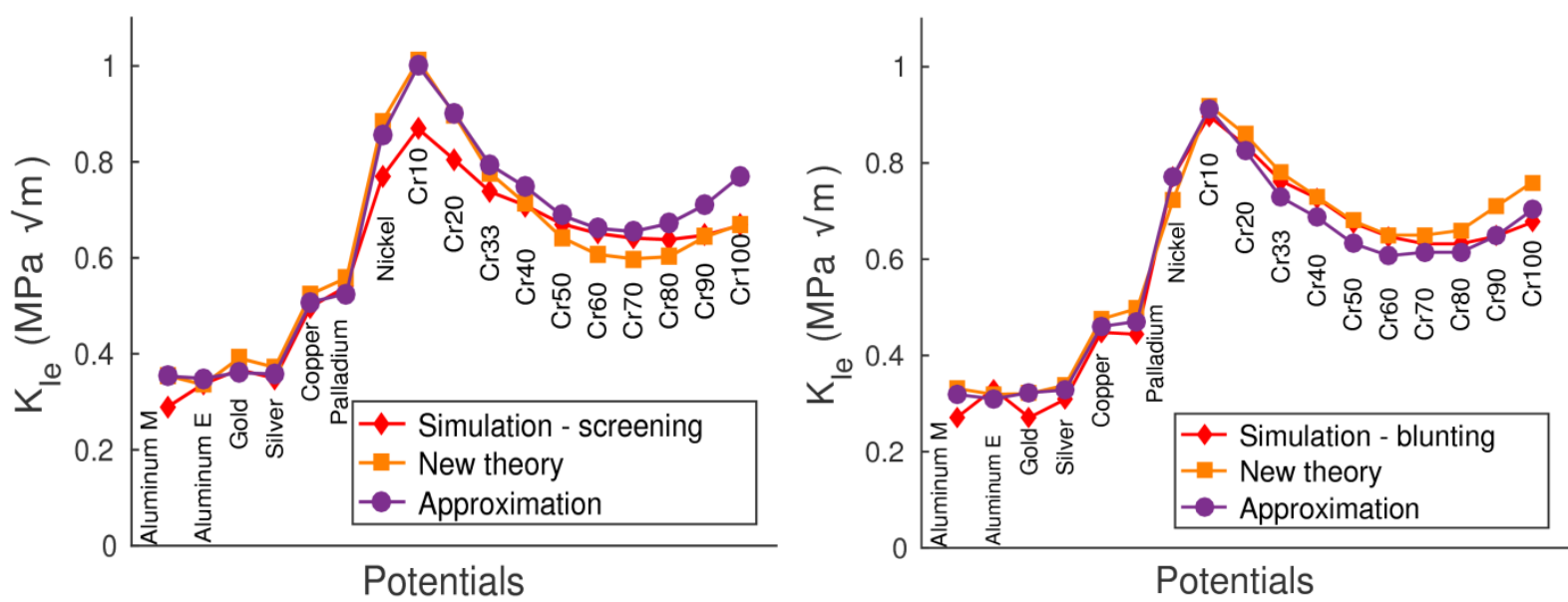

Figure 15: Critical stress intensity factor for dislocation emission for all materials studied here; Simulations (red), full theory (orange); approximate model (purple) for a) screening and b) blunting.

The above approximation still requires computation of $\Psi_{n u c}(\Delta)$ for the specific crack tip geometry. A simpler model that depends only on the unstable stacking fault energy $\gamma_{u s f}$ and surface energy $\gamma_{s}$ is very valuable since these quantities are easily computed via firstprinciples methods using simple periodic-cell geometries. We first recall that $\gamma_{\text {step }} \approx 0.7 \gamma_{s}$. Then, the values $\Delta_{1}^{c}=0.36 b_{p}, \Delta_{2}^{c} / \Delta_{1}^{c} \approx 0.7$ (and so $\Delta_{2}^{c}=0.25 b_{p}$ ) capture both "screening" and "blunting" well, for most of the studied materials.

560 From Eq. 12 and a simple Peierls model, the contribution from the GSF energy is then $\Psi_{g s f}\left(0.25 b_{p}\right)=0.5 \gamma_{u s f}$. Again using a simple Peierls model, the contribution from the crack tip unit is $\Psi_{n u c}\left(0.36 b_{p}\right)-\Psi_{\text {nuc }}\left(0.25 b_{p}\right)=0.7 \gamma_{\text {step }}-0.5 \gamma_{\text {step }}=0.14 \gamma_{s}$. Therefore, a good analytic estimate of the critical energy release rate at the point of dislocation emission is $G_{I e}=0.14 \gamma_{s}+0.5 \gamma_{u s f}$. A small correction to this estimate precisely captures the average $G_{I e}$ across the entire set of simulations,

$$
G_{I e}=0.145 \gamma_{s}+0.5 \gamma_{u s f}
$$

Eq. 14 applies to systems with high step energies (the dominant case in real materials). The full model reduces to the Rice model as the step energy decreases, $K_{I e} \rightarrow K_{I e, R i c e}$ as $\gamma_{\text {step }} \rightarrow \gamma_{u s f}$ with $\gamma_{s} \approx \gamma_{u s f} / 0.7$ (see Peierls analysis and Table 2). Eq. 14 does not capture this limit, and should not be used when the surface energy/step energy are small. An analytic model that captures the correct limit is thus 
This is a post-print of the following article: Andric, Predrag; Curtin, W. A. Journal Of The Mechanics And Physics Of Solids $2017,23$. 315-337.. The formal publication is available at http://dx.doi.org/10.1016/j.jmps.2017.06.006 @ 2017. This manuscript version is made available under the CC-BY-NC-ND 4.0 license http://creativecommons.org/licenses/by-nc-nd/4.0/

$$
\begin{aligned}
& G_{I e}=0.145 \gamma_{s}+0.5 \gamma_{u s f}, \quad \gamma_{s}>3.45 \gamma_{u s f} \\
& G_{I e}=\gamma_{u s f}, \quad \gamma_{s}<3.45 \gamma_{u s f}
\end{aligned}
$$

575 Figure 16 shows the predictions of Eq. 15 for $G_{I e}$ along with the simulation results (screening and blunting). Also included are additional simulations and predictions for a family of pairpotentials having fixed $\gamma_{s}$ and varying $\gamma_{u s f}$ [28], using full anisotropy not included in Ref. [28]. The agreement is very good across the entire spectrum of materials. The single analytical formula of Eq. 15, with $K_{\text {Ie }}$ following from Eq. 13, is thus a suitable analytic replacement for the Rice model that incorporates the effect of the step energy and is based on a deeper understanding of the crack tip processes controlling dislocation nucleation.

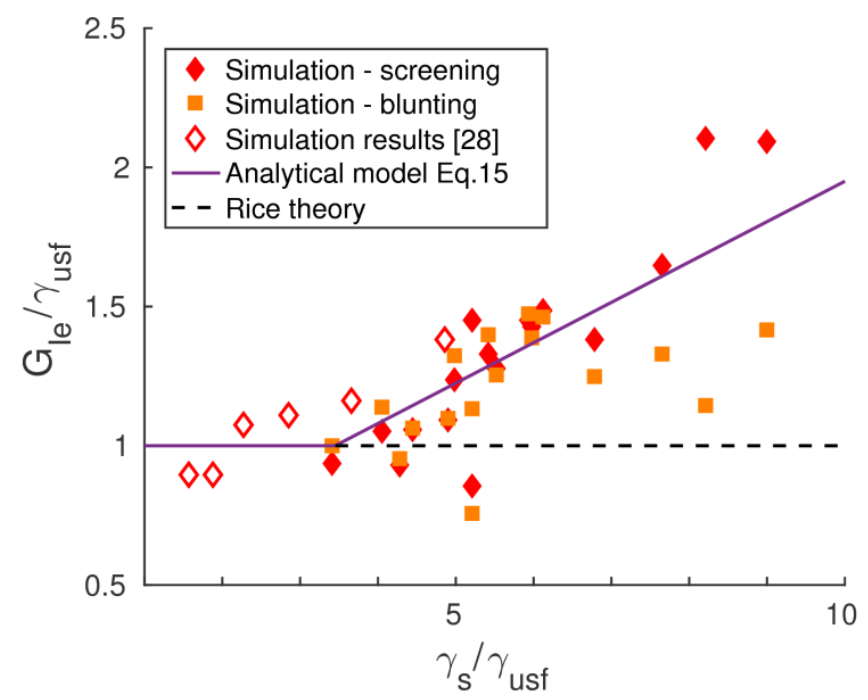

Figure 16: Critical energy release rate $G_{I e}$ as a function of the materials surface energy $\gamma_{\mathrm{s}}$ normalized by $\gamma_{\text {usf }}$ for all materials studied here; Simulations - screening (red diamonds), Simulations - blunting (orange squares), Simulation results from [28] (open red diamonds), Analytical model Eq. 15 (purple line); Rice theory (black dashed line).

\section{Discussion}

The Rice theory is an elegant, long-standing, and well-accepted model for the approximate analysis of dislocation emission at a crack tip under Mode I loading. However, we have shown that a new model is necessary for three important reasons. First, the Rice theory predictions for $K_{I e}$ and $\Delta_{1}^{c}$ are inconsistent with detailed molecular simulations: the predicted $K_{I e}$ is too low while the predicted $\Delta_{1}^{c}$ is too high, and these two deviations cannot be reconciled within the context of the Rice theory. Second, the Rice theory neglects entirely the energy associated with formation of the surface step upon emission, and simulations demonstrate clear that such a step exists and that the energy cost is high compared to the 
This is a post-print of the following article: Andric, Predrag; Curtin, W. A. Journal Of The Mechanics And Physics Of Solids $2017,23$. 315-337.. The formal publication is available at http://dx.doi.org/10.1016/j.jmps.2017.06.006 @ 2017. This manuscript version is made available under the CC-BY-NC-ND 4.0 license http://creativecommons.org/licenses/by-nc-nd/4.0/

unstable stacking fault energy. Third, explicit computation of the energy versus shear displacement at the crack tip shows no energy maximum, yet a maximum is required in the Rice analysis since the maximum sets the point of emission.

The new theory is based on the recognition that the crack tip structural unit is impeded from shearing by the energy cost of the step. The nascent dislocation is thus "trapped" by the lattice and can only be emitted when the crack tip structural unit reaches a point of mechanical instability. This instability is fundamentally different from the Rice concept, which envisions a continuous cohesive zone behaviour with no "trapping". We have demonstrated the physical behaviour, and rationalized the simulation results, using a Peierls model for the nucleation energy at the crack tip and for the generalized stacking fault energy along the remainder of the slip plane. We can then compute the critical energy release rate at the emission instability, from which we can find $K_{I e}$. The simple Peierls model produces the trends seen in simulations and reduces to the Rice model when the step energy is zero. We have shown that the new theory, a simplified approximation, and an analytic model (Eq. 15), are in very good agreement with simulation results across 17 different fcc materials (interatomic potentials).

Several extensions of the present analysis are necessary. First, dislocation nucleation is a thermally activated process at finite temperatures. Computing the energy barrier for nucleation at loads $K_{I}<K_{I e}$ is required, and the step energy will contribute an important component to this energy barrier. Second, further dislocation emission events are important, especially the second partial emission that determines whether there is crack tip twinning or formation of a full dislocation that can glide away from the crack tip [29]. The transition from 615 twinning partial emission to trailing partial emission at finite $\mathrm{T}$ is controlled by thermal activation and the Rice theory is not fully consistent with simulations [29, 30]. We will report on these issues in future work.

The new theory has implications for chemical effects on dislocation nucleation. Chemical species adsorbed at the crack tip can change the step energy, and thus alter the load needed for dislocation emission. The Rice theory accommodates chemistry only through the influence of chemical species on $\gamma_{u s f}$. Based on the ratio $\gamma_{u s f} / \gamma_{s}=0.18 \pm 0.05$ for materials studied in this work, the current theory shows that the step energy contributes $\sim 65 \%$ of the critical energy release rate, and hence will predict different trends for emission versus specific chemical environment then the standard model. The role of local chemical transport at the crack tip, and hence kinetic effects, then also becomes important in determining 
This is a post-print of the following article: Andric, Predrag; Curtin, W. A. Journal Of The Mechanics And Physics Of Solids $2017,23$. 315-337.. The formal publication is available at http://dx.doi.org/10.1016/j.jmps.2017.06.006 @ 2017. This manuscript version is made available under the CC-BY-NC-ND 4.0 license http://creativecommons.org/licenses/by-nc-nd/4.0/

emission just as in cleavage [31]. There is some experimental evidence for hydrogenenhanced dislocation emission that may be consistent with effects predicted by extension of the present model [32] while coupled Quantum/Continuum methods show that the precise position of an $\mathrm{H}$ impurity relative to the slip plane determines whether $K_{I e}$ is increased or decreased [36]. Thus, behaviour at the step will influence chemical embrittlement, i.e. the transition from emission (ductile behaviour) to cleavage (brittle behaviour) in the presence of a chemical environment around the crack.

The present analysis fundamentally changes the dependence of the ductile-to-brittle transition on underlying material properties. A material is brittle when $K_{I c}<K_{I e}$. The standard analyses use the Griffith model $K_{I C}=\sqrt{2 \gamma_{S} o^{\prime}}$, where $o^{\prime}$ is a material property for Griffith cleavage under mode I and use the Rice model $K_{I e}=\sqrt{\gamma_{u s f} O(\theta, \varphi)} / F(\theta)_{12}$. The new analysis here shows that $\gamma_{s, e}$ (surface energy along the emission plane) also enters into the emission criterion. Using our simple analytic model (Eq. 15), the analysis predicts $K_{I e}=$ $\sqrt{\left(0.5 \gamma_{u s f}+0.145 \gamma_{s, e}\right) o(\theta, \varphi)} / F(\theta)_{12}$ for $\gamma_{s} / \gamma_{u s f}>3.45$. Thus, decreasing the surface energies of a material decreases both $K_{I c}$ and $K_{I e}$, making embrittlement less likely.

The new model recently resolves a discrepancy found between molecular simulations and predictions of the standard brittle/ductile analysis in $\mathrm{Mg}$ [34]. In the standard analysis, cases involving slip along the basal plane of hcp $\mathrm{Mg}$ are predicted to emit dislocations $\left(K_{I c}>\right.$ $K_{I e}$ ) but which are observed to cleave in simulations. Although $\mathrm{Mg}$ has an hep crystal structure, with a different elastic anisotropy, and although the fracture planes and slip planes differ, the present model remains applicable. Here, we apply the analytic model of Eq. 15 to compute $K_{I e}$ including the surface energy cost along the slip plane. The necessary energies are reproduced in Table 3. Predictions of $K_{I e}$ using the Rice analysis (see Ref. [34]) and the present model (Eq. 15) are also shown in Table 3, along with the simulation results for sharp cracks. In contrast to the standard analysis, the new model now correctly predicts all the observed cleavage cases $K_{I c} \leq K_{I e}$, and retains the previous prediction of emission for the case where $K_{I c}$ is only slightly larger than $K_{I e}$. Eq. 15 remains imperfect, and the competition between cleavage and emission in $\mathrm{Mg}$ is rather subtle, but nonetheless the incorporation of the surface/step energy into the analysis leads to predictions that are consistent with 655 simulations. This demonstrates that the model is valuable for better assessment of brittle vs. ductile behaviour. 
This is a post-print of the following article: Andric, Predrag; Curtin, W. A. Journal Of The Mechanics And Physics Of Solids $2017,23$. 315-337.. The formal publication is available at http://dx.doi.org/10.1016/j.jmps.2017.06.006 @ 2017. This manuscript version is made available under the CC-BY-NC-ND 4.0 license http://creativecommons.org/licenses/by-nc-nd/4.0/

\begin{tabular}{|c|c|c|c|c|c|c|c|c|c|c|c|}
\hline $\begin{array}{c}\text { Crack } \\
\text { plane }\end{array}$ & $\begin{array}{l}\text { Orientation } \\
\text { (n) }[1]\end{array}$ & $\begin{array}{c}\gamma_{\mathrm{s}} \\
\left(\mathrm{mJ} / \mathrm{m}^{2}\right)\end{array}$ & $\begin{array}{l}\text { Slip } \\
\text { plane }\end{array}$ & $\begin{array}{c}\gamma_{\mathrm{s}, \mathrm{e}} \\
\left(\mathrm{mJ} / \mathrm{m}^{2}\right)\end{array}$ & $\underset{\left(\mathrm{mJ} / \mathrm{m}^{2}\right)}{\gamma_{\mathrm{usf}}}$ & $\begin{array}{c}\mathrm{K}_{\mathrm{Ic}} \\
\left(\mathrm{MPa} \mathrm{m}^{1 / 2}\right)\end{array}$ & $\begin{array}{c}\mathrm{K}_{\mathrm{Ie}, \text { Rice }} \\
\left(\mathrm{MPa} \mathrm{m}^{1 / 2}\right)\end{array}$ & $\begin{array}{c}\mathrm{K}_{\mathrm{Ie}, \text { new }} \\
\left(\mathrm{MPa} \mathrm{m}^{1 / 2}\right)\end{array}$ & $\begin{array}{c}\text { Rice } \\
\text { prediction }\end{array}$ & $\begin{array}{c}\text { New theory } \\
\text { prediction }\end{array}$ & $\begin{array}{c}\mathrm{MD} \\
\text { results }\end{array}$ \\
\hline Prism I & $(\overline{1} 010)[1 \overline{2} 10]$ & 582 & Basal & 568 & 125 & 0.252 & 0.236 & 0.254 & Emission & Cleavage & Cleavage \\
\hline Prism II & $(\overline{1} 2 \overline{1} 0)[\overline{1} 010]$ & 651 & Basal & 568 & 125 & 0.267 & 0.262 & 0.282 & Emission & Cleavage & Cleavage \\
\hline Pyramidal I & $(10 \overline{1} 0)[1 \overline{2} 10]$ & 619 & Basal & 568 & 125 & 0.262 & 0.222 & 0.239 & Emission & Emission & Emission \\
\hline Pyramidal II & $(11 \overline{2} 2)[\overline{1} 010]$ & 647 & Basal & 568 & 125 & 0.269 & 0.250 & 0.269 & Emission & Cleavage & Cleavage \\
\hline
\end{tabular}

Table 3: Crack tip cleavage/emission competition in magnesium as predicted from (i) Rice theory, (ii) The new theory for dislocation emission, and (iii) as observed in atomistic simulations. The values of material properties and simulations results are taken from [34].

In summary, a new model for emission based on a local crack tip mechanical instability has been shown to explain, conceptually and quantitatively, the crack tip emission process including the role of step formation at the crack tip. The model has been painstakingly validated across a wide range of fcc materials. Of great practical importance, we have provided an accurate analytic model (Eq. 15) that requires only easily-computable material parameters as input. In addition to its new insights into the physical origin of dislocation emission, this model provides a path for (i) designing new materials that exhibit the desired ductile behaviour (dislocation emission), which is a necessary precursor to ductile fracture, and (ii) better understanding of chemical embrittlement due to a change in crack tip behaviour from emission (ductile) to cleavage (brittle) behaviour. We will report on research examining these and other implications in the near future.

Acknowledgements: This work was supported by the European Research Council through the Advanced Grant “Predictive Computational Metallurgy", ERC Grant agreement No.339081 PreCoMet. 
This is a post-print of the following article: Andric, Predrag; Curtin, W. A. Journal Of The Mechanics And Physics Of Solids $2017,23$. 315-337.. The formal publication is available at http://dx.doi.org/10.1016/j.jmps.2017.06.006 ( ) 2017. This manuscript version is made available under the CC-BY-NC-ND 4.0 license http://creativecommons.org/licenses/by-nc-nd/4.0/

\section{Appendix A. Anisotropic elastic properties}

Calculation of the material elastic property $o(\varphi, \theta)$, based on the Stroh formalism [16] is given by

$$
o(\phi, \theta)=s_{i}(\phi) \Lambda_{i j}^{(\theta)^{-1}} s_{j}(\phi)
$$

685

where $s(\varphi)$ is a slip vector in the constrained path approximation and

$$
\Lambda_{i j}^{(\theta)}=\Omega_{i k} \Lambda_{k l} \Omega_{l j}
$$

$\Lambda_{i j}$ is the appropriate matrix for crack orientation and $\Omega_{i j}$ is rotation matrix given by

$$
\Omega=\left[\begin{array}{ccc}
\cos \theta & \sin \theta & 0 \\
-\sin \theta & \cos \theta & 0 \\
0 & 0 & 1
\end{array}\right]
$$

For an atomically sharp, semi-infinite crack in homogeneous materials $\Lambda_{i j}$ is given by

$$
\Lambda=\frac{1}{2} L^{-1}
$$

where

$$
L^{-1}=\operatorname{Re}\left(i A B^{-1}\right) .
$$

In equation $\mathrm{A} .5, \mathrm{~A}$ and $\mathrm{B}$ are eigenvector matrices given by

$$
\begin{gathered}
N \xi=p \xi \\
N=\left[\begin{array}{ll}
N_{1} & N_{2} \\
N_{3} & N_{1}^{T}
\end{array}\right], \xi=\left[\begin{array}{l}
A \\
B
\end{array}\right]
\end{gathered}
$$

where

$$
N_{1}=-T^{-1} R^{T}, \quad N_{2}=T^{-1}, N_{3}=R T^{-1} R^{T}-Q
$$

and

$$
Q_{i k}=C_{i 1 k 1}, R_{i k}=C_{i 1 k 2,}, T_{i k}=C_{i 2 k 2}
$$

In the last equation $C_{i j k 1}$ is material stiffness tensor.

The angular distribution of displacements near a crack tip depend on material elastic constants via $p_{l}$, $p_{2}, q_{1}$ and $q_{2}$ in anisotropic materials. These constants can be found from

$$
\begin{aligned}
& p_{1}=S_{11}^{p} a_{1}^{2}+S_{12}^{p}-S_{16}^{p} a_{1} \\
& p_{2}=S_{11}^{p} a_{2}^{2}+S_{12}^{p}-S_{16}^{p} a_{2} \\
& q_{1}=S_{12}^{p} a_{1}+S_{22}^{p} / a_{1}-S_{26}^{p} \\
& q_{2}=S_{12}^{p} a_{2}+S_{22}^{p} / a_{2}-S_{26}^{p}
\end{aligned}
$$

where $S_{i j}^{p}$ are members of the compliance matrix for $2 \mathrm{D}$ plane strain problems when $\mathrm{x}-\mathrm{y}$ is the plane of symmetry, and $a_{1}=\alpha_{1}+i \beta_{1}$ and $a_{2}=\alpha_{2}+i \beta_{2},\left(\beta_{1}, \beta_{2}>0\right)$, are the roots of the following characteristic equation

$$
S_{11}^{p} a^{4}-2 S_{16}^{p} a^{3}+\left(2 S_{12}^{p}+S_{66}^{p}\right) a^{2}-2 S_{26}^{p} a+S_{22}^{p}=0 .
$$

The entries of the compliance matrix for plane strain problems can be found from

$$
S_{i j}^{p}=S_{i j}-\frac{S_{i 3} S_{3 j}}{S_{33}}
$$

and $S_{i j}$ are members of the material compliance matrix. For more details see [35]. 


\begin{tabular}{|c|c|c|c|c|c|c|c|c|c|}
\hline Material & $\mathrm{a}(\AA)$ & $\mathrm{b}_{\mathrm{p}}(\AA)$ & $\mathrm{C}_{11}(\mathrm{GPa})$ & $\mathrm{C}_{12}(\mathrm{GPa})$ & $\mathrm{C}_{44}(\mathrm{GPa})$ & $\mathrm{o}\left(\theta=70^{\circ}, \phi=0\right)(\mathrm{GPa})$ & $\mathrm{o}^{\prime}(\mathrm{GPa})$ & $\gamma_{\mathrm{usf}}\left(\mathrm{J} / \mathrm{m}^{2}\right)$ & $\gamma_{\mathrm{s}}\left(\mathrm{J} / \mathrm{m}^{2}\right)$ \\
\hline Aluminum M [19] & 4.050 & 1.653 & 113.4 & 61.5 & 31.6 & 87.9 & 88.7 & 0.167 & 0.871 \\
\hline Aluminum E [20] & 4.032 & 1.646 & 118.0 & 62.2 & 36.7 & 97.0 & 98.2 & 0.119 & 0.871 \\
\hline Gold [22] & 4.080 & 1.666 & 183.2 & 158.7 & 45.3 & 101.0 & 103.7 & 0.097 & 0.796 \\
\hline Silver [22] & 4.090 & 1.670 & 129.1 & 91.7 & 56.7 & 115.4 & 120.0 & 0.119 & 0.619 \\
\hline Copper [21] & 3.615 & 1.476 & 169.9 & 122.6 & 76.2 & 152.1 & 158.4 & 0.162 & 1.240 \\
\hline Palladium [22] & 3.890 & 1.588 & 221.1 & 183.0 & 72.6 & 152.2 & 157.4 & 0.145 & 1.301 \\
\hline Nickel [19] & 3.520 & 1.437 & 247.9 & 147.8 & 124.8 & 249.8 & 260.0 & 0.368 & 1.631 \\
\hline Cr10 [24] & 3.497 & 1.428 & 301.3 & 171.5 & 156.7 & 311.2 & 324.1 & 0.425 & 1.445 \\
\hline Cr20 [24] & 3.507 & 1.432 & 276.6 & 165.6 & 148.7 & 287.5 & 300.4 & 0.356 & 1.441 \\
\hline $\mathrm{Cr} 33$ [24] & 3.522 & 1.438 & 246.6 & 158.1 & 138.5 & 257.8 & 270.5 & 0.288 & 1.434 \\
\hline Cr40 [24] & 3.529 & 1.441 & 232.6 & 154.7 & 133.7 & 243.7 & 256.3 & 0.264 & 1.430 \\
\hline Cr50 [24] & 3.541 & 1.446 & 213.1 & 150.8 & 127.6 & 223.7 & 236.2 & 0.240 & 1.424 \\
\hline Cr60 [24] & 3.552 & 1.450 & 205.5 & 150.3 & 124.6 & 214.7 & 227.1 & 0.232 & 1.418 \\
\hline Cr70 [24] & 3.563 & 1.454 & 204.5 & 152.8 & 124.6 & 212.4 & 224.8 & 0.237 & 1.413 \\
\hline Cr80 [24] & 3.572 & 1.458 & 211.2 & 159.0 & 127.9 & 218.1 & 230.8 & 0.255 & 1.408 \\
\hline Cr90 [24] & 3.579 & 1.461 & 225.6 & 168.9 & 134.9 & 231.8 & 245.2 & 0.287 & 1.404 \\
\hline Cr100 [24] & 3.584 & 1.463 & 247.3 & 182.5 & 145.5 & 253.3 & 267.5 & 0.328 & 1.400 \\
\hline
\end{tabular}

Table 4: Material parameters used for investigation of a crack tip dislocation emission 
This is a post-print of the following article: Andric, Predrag; Curtin, W. A. Journal Of The Mechanics And Physics Of Solids $2017,23$. 315-337.. The formal publication is available at http://dx.doi.org/10.1016/j.jmps.2017.06.006 @ 2017. This manuscript version is made available under the CC-BY-NC-ND 4.0 license http://creativecommons.org/licenses/by-nc-nd/4.0/

\section{Appendix B. Shearing of the triangular crack tip structural unit}

In the presented theoretical model we analyze the shear deformation of triangular structural units along the slip plane. The crack tip dislocation emission is controlled by the local mechanical instability at the crack tip, which is achieved by the critical shear displacement of the crack tip structural unit. The new theory assumes that triangular structural units along the slip plane (see Figure 17a) are subjected to pure shear deformation. If the structural unit is subjected to pure shear, it will change the shape but not the area (see Figure 17b). Then, the relative shear displacement of the crack tip unit can be computed as $\Delta_{1}=u_{1}^{\prime}-u_{0}=u_{1}^{\prime}-u_{1}=\Delta_{1}^{\prime}$, where $u_{i}$ is the atom displacement in [112] direction. This is the case when we compute the nucleation energy, and in Mode II simulations (with some small deviations). In Mode I crack simulations, the K-field introduces normal stress parallel to the slip plane. Due to normal stress in the [112] direction, the area of the structural units will be changed (see Figure 17c). This is an additional energy which is not incorporated in the standard nucleation energy. Also, the additional displacement causes that $\Delta_{1} \neq \Delta_{1}^{\prime}$. This can make a confusion which relative displacement is the representative one. In studied Mode I simulations, the Y coordinate of atoms 1 and 1' is the same, $Y_{1}=Y_{1}$, (see the initial geometry in Figure 4). Due to this geometry, we conclude that dislocation emission is controlled by $\Delta_{1}$ relative shear displacement. One can also use $\Delta_{1}$, , or an average value, as a measure of the crack tip displacement and he will find that predicted results are slightly higher than what we presented (Al M, Al E, Au, Ag, Cu, Pd and Ni potentials). The bigger discrepancy can arise if the used crack geometry changes the vertical position of the crack plane. This is what we observe with $\mathrm{Cr} 10-\mathrm{Cr} 100$ potentials and this effect is caused by the surface relaxation. Due to the mentioned effect, in the blunting case we use $\Delta_{1}$ ' shear displacement for the same potentials. The additional stretching of the structural units is present along the slip plane. We think that this effect is important as the opening softening, even though so far it was not explained, nor even commented.
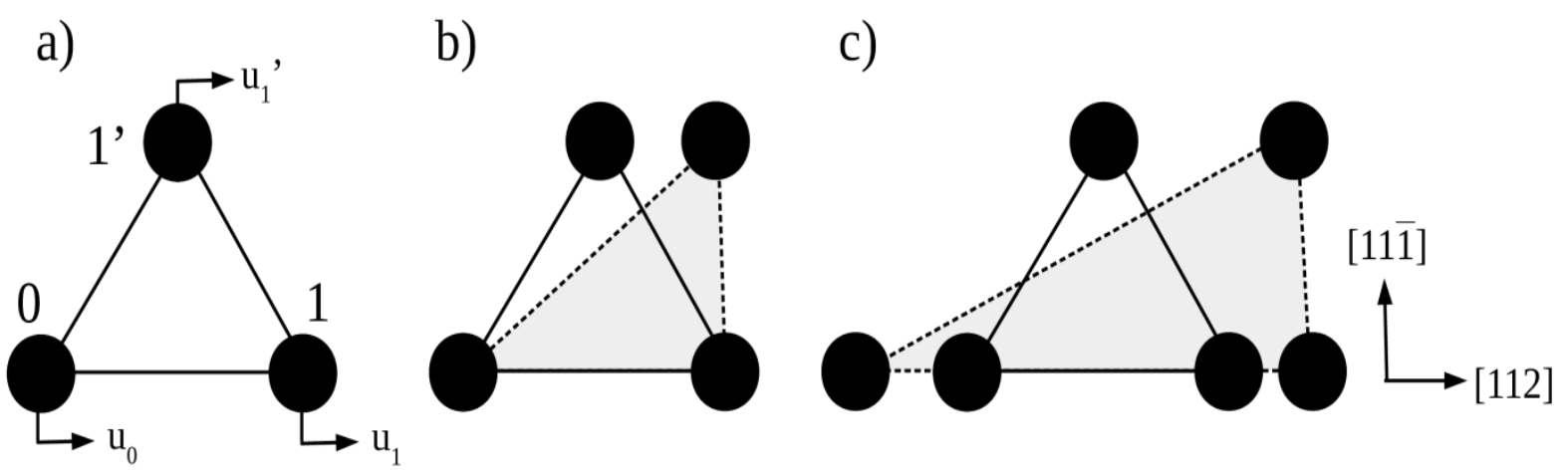

Figure 17: Studied crack tip triangular structural unit: a) initial geometry; b) the crack tip unit under pure shear; c) the crack tip unit under shear and normal stresses.

\section{Appendix C. Comparison of shear displacement profiles in Mode I and Mode II}

Both the Rice theory and the new theory assume that the shear displacement distribution along the slip plane for Mode I, caused by $K_{I I}^{e f f}=K_{I} F_{12}(\theta)$, is the same as that for Mode II. In the new theory, the only difference is that the shear displacement of the crack tip unit is controlled by the nucleation energy rather than the GSF energy. Therefore, the same crack tip shear displacement $\Delta_{1}$ is achieved at different applied $\mathrm{K}$ values for Mode I and Mode II. Nonetheless, for a given crack tip shear displacement $\Delta_{1}$, the shear displacements $\Delta_{2}$, 
This is a post-print of the following article: Andric, Predrag; Curtin, W. A. Journal Of The Mechanics And Physics Of Solids $2017,23$. 315-337.. The formal publication is available at http://dx.doi.org/10.1016/j.jmps.2017.06.006 ( ) 2017. This manuscript version is made available under the CC-BY-NC-ND 4.0 license http://creativecommons.org/licenses/by-nc-nd/4.0/

$\Delta_{3}, \ldots$. should be the same in Mode I and Mode II. We have verified this explicitly via simulation. Specifically, we measure the shear displacements $\Delta_{i}(\mathrm{i}=1,2,3, \ldots)$ along the slip plane for both increasing Mode II $\mathrm{K}_{\text {II }}$ and increasing Mode I $\mathrm{K}_{\mathrm{I}}$, and obtain the displacements $\Delta_{i, I I}\left(K_{I I}\right)$ and $\Delta_{i, I}\left(K_{I I}^{e f f}\right)$. We then find the load levels $\mathrm{K}_{\mathrm{II}}$ and $K_{I I}^{\text {eff }}$ at which the crack tip shear displacements are equal, $\Delta_{1, I I}\left(K_{I I}\right)=\Delta_{1, I}\left(K_{I I}^{\text {eff }}\right)$, and examine the shear displacements $\Delta_{i, I I}\left(K_{I I}\right)$ and $\Delta_{i, I}\left(K_{I I}^{e f f}\right)$ for $\mathrm{i}=2,3 \ldots$ further along the slip plane. The results for Ni are shown in Figure 18, where we show the "screening" case for Mode II and the "blunting" case for Mode I so as to add an extra apparent level of difference. Figure 18 demonstrates that the slip distribution along the slip plane is essentially identical for Mode I and Mode II loadings at the same crack tip displacement $\Delta_{1}$. The step arising in the Mode I case restrains the crack tip shear displacement $\Delta_{1, I}$, and so a larger applied $K_{I I}^{e f f}$ is required to obtain the same displacement that would be obtained in Mode II (or in Mode I with no step). This restraint is seen in the graphical analysis of Figure 11.

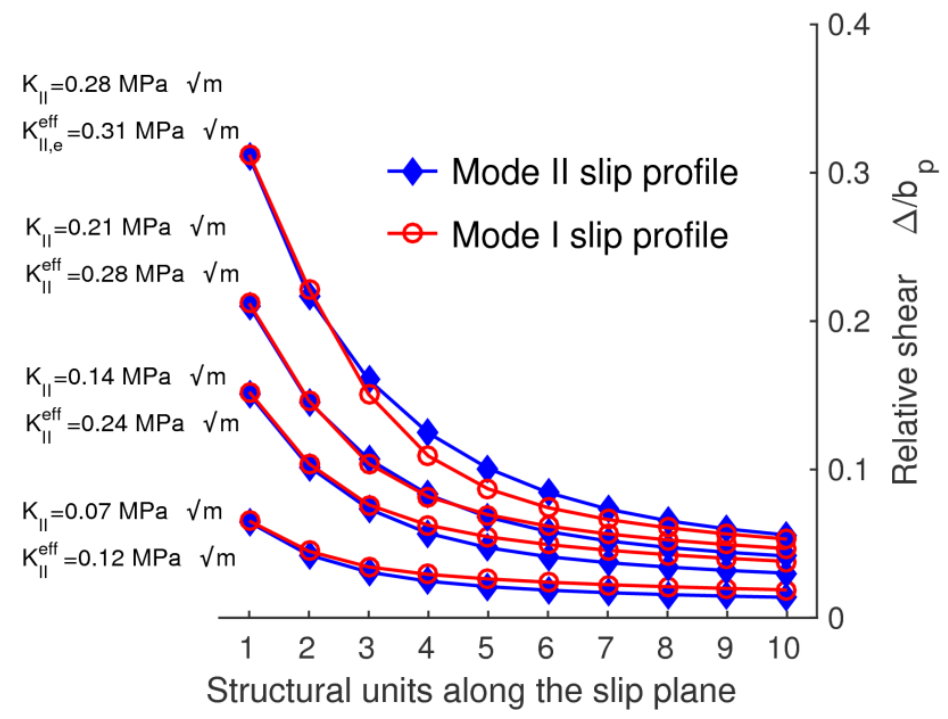

Figure 18: The shear displacement distribution along the slip plane in Mode II (screening) and Mode I (blunting) at different farfield $\mathrm{K}$ in $\mathrm{Ni}$.

\section{Appendix D. Computation of the crack-tip parameter $\beta$}

The crucial parameter in describing the crack tip displacement is parameter $\beta$. Here we present an approximate computation method for finding the necessary parameter. As mentioned in Appendix C, at same applied far-field loading we observe different crack tip displacements depending on the step presence. The surface step creation changes the shear resistance of the crack tip structural unit. Within known shear displacements of crack tip unit at same far-field loading $K_{I I}=K_{I I}^{e f f}$, and with known restoring stresses, the slope of the applied stress $\tau_{\text {app }}$ is defined. Using this approach we compute the parameter $\beta$ for 10 studied fcc materials. Results are given in Table 5 and as we expected the values are between 1.4 and 2.3. The surprisingly low value we find in gold which can be due to high material anisotropy. Figure 19 shows the family of the applied stresses computed in Nickel along with the restoring stresses. Our atomistic simulations reveal that applied stresses $\tau_{a p p}$ are indeed parallel when effects due to non-linearity are negligible. 
This is a post-print of the following article: Andric, Predrag; Curtin, W. A. Journal Of The Mechanics And Physics Of Solids $2017,23$. 315-337.. The formal publication is available at http://dx.doi.org/10.1016/j.jmps.2017.06.006 @ 2017. This manuscript version is made available under the CC-BY-NC-ND 4.0 license http://creativecommons.org/licenses/by-nc-nd/4.0/

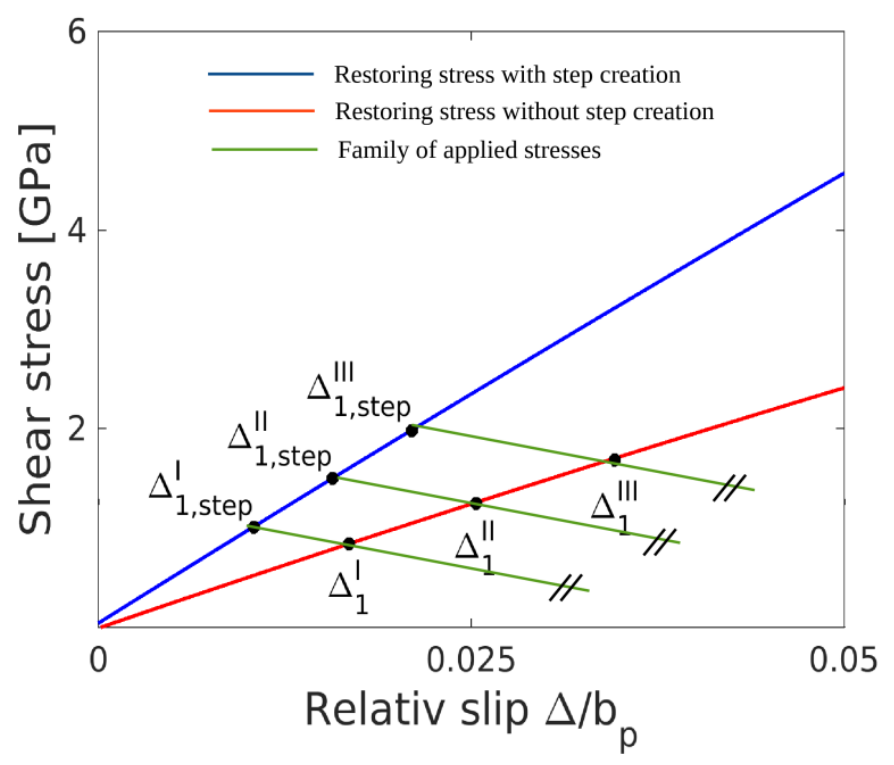

Figure 19: Restoring stress of the crack tip structural with the surface step creation (the blue line) and without the surface step creation (the red line) and the family of applied stresses (green lines) for different applied $\mathrm{K}_{\mathrm{I}}$ in Nickel.

\begin{tabular}{|c|c|}
\hline Element & Parameter $\boldsymbol{\beta}$ \\
\hline $\mathrm{Cr} 100$ & 1.91 \\
$\mathrm{Cr} 50$ & 2.26 \\
$\mathrm{Cr} 10$ & 2.36 \\
$\mathrm{Al} \mathrm{M}$ & 1.83 \\
$\mathrm{AlE}$ & 1.48 \\
$\mathrm{Au}$ & 1.23 \\
$\mathrm{Ag}$ & 1.4 \\
$\mathrm{Cu}$ & 2.62 \\
$\mathrm{Ni}$ & 2.15 \\
$\mathrm{Pd}$ & 1.41 \\
\hline
\end{tabular}

Table 5: Values of the parameter $\beta$ computed in various fcc systems.

\section{Appendix E. Assessment of the theory for different slip plane orientations}

The new theory presented in the paper, and the Rice theory as well, assume that dislocation emission from a crack tip in Mode I is controlled by the effective mode II stress intensity factor $K_{I I}^{\text {eff }}$. All differences with respect to the slip plane inclination angle $\theta$ are accounted for in $K_{I I}^{e f f}$. Here, we examine this assumption in all respects using molecular statics crack simulations at $\theta=35.3^{0}, 54.7^{0}, 70.5^{\circ}$, and $90^{\circ} \mathrm{in}$ fcc $\mathrm{Ni}$.

First, we show that a slip profile along the sliding plane is independent of the slip plane inclination angle $\theta$. For each inclination angle we find the far field $\mathrm{K}_{\mathrm{I}}$ loading that causes the same shear displacement of the crack tip unit $\Delta_{1}^{i}(i=I, I I, I I I, \ldots)$ at all orientation angles. We then compare the shear displacements of the structural units further along the slip plane $\Delta_{j}^{i}(j=2,3,4, \ldots)$. We find that the slip profiles along the sliding plane are indeed independent of the inclination angle, as shown in Figure 20a. At the highest testing load, there is a very small deviation along the sliding plane when $\theta=35.3^{0}$ which could be, at this angle, due to the some non-linear effects caused by the larger opening displacement along the sliding plane.

Second, we compare $\mathrm{K}_{I I}^{e f f}=K_{I} F_{12}(\theta)$ for the three crack tip shear displacements $\Delta_{1}^{i}(i=I, I I, I I I)$ at each angle, as shown in Figure 20b. $K_{I I}^{\text {eff }}$ is nearly independent of $\theta$, but does show a decreasing trend with increasing $\theta$. This could be due to some softening effects caused by the normal stresses (normal and parallel to the sliding plane). 
This is a post-print of the following article: Andric, Predrag; Curtin, W. A. Journal Of The Mechanics And Physics Of Solids $2017,23$. 315-337.. The formal publication is available at http://dx.doi.org/10.1016/j.jmps.2017.06.006 @ 2017. This manuscript version is made available under the CC-BY-NC-ND 4.0 license http://creativecommons.org/licenses/by-nc-nd/4.0/

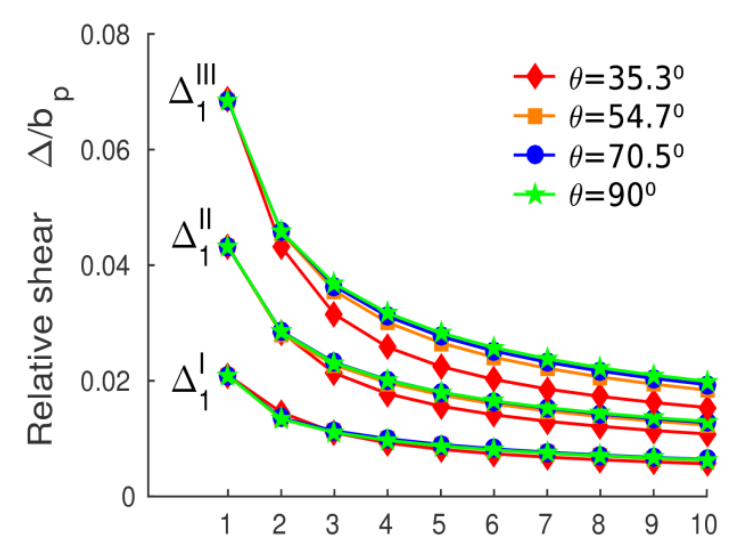

Structural units along the slip plane

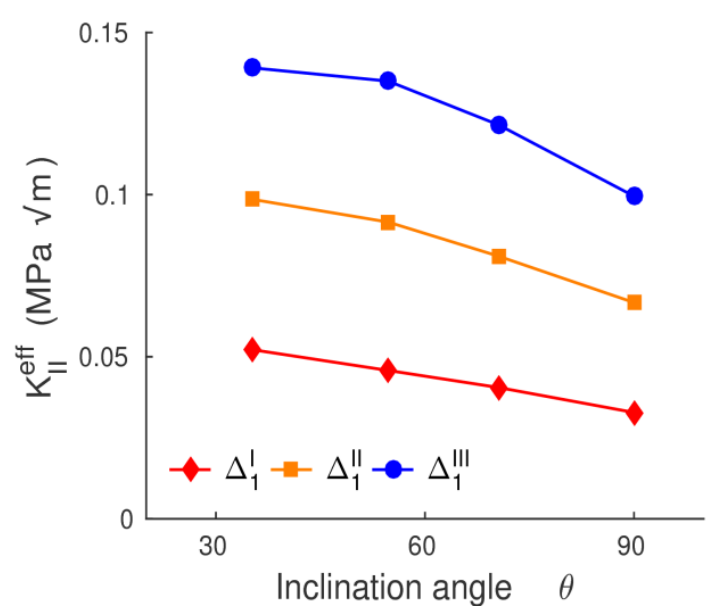

Figure 20: a) The shear displacement distribution along the slip plane in Mode I for different inclination angles $\theta$; b) The effective Mode II stress intesity factor $\mathrm{K}_{I I}^{e f f}$ as a function of the inclination angle $\theta$ which causes the same crack tip shear displacements.

Third, we examine the nucleation energy including the surface step for the different inclination angles. The nucleation energy as a function of the relative slip $\Psi_{n u c}(\Delta)$ is computed for different inclination angles using the computational method described in Section IV. As shown in Figure 21, the energy and slip resistance are indeed independent of angle for $\theta \geq 54.7^{\circ}$ and show only small deviations for $\theta=35.3^{\circ}$.
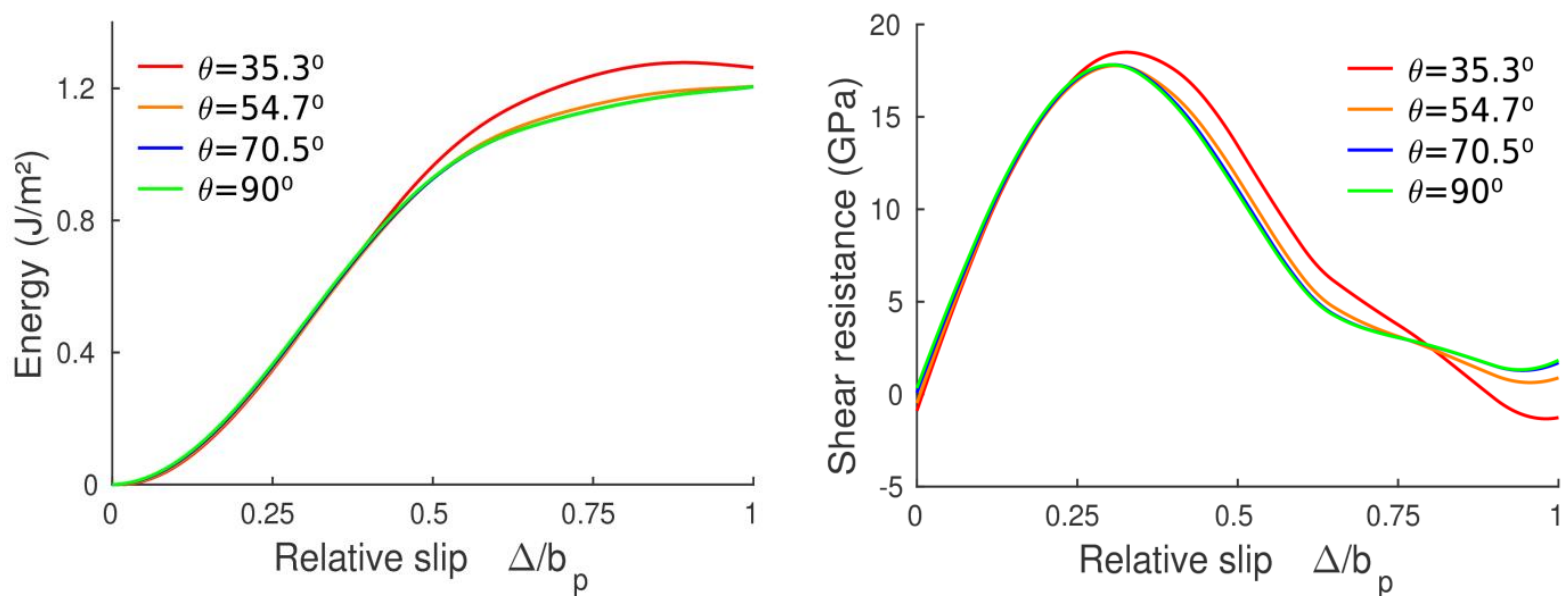

Figure 21: a) Nucleation energy as a function of the relative slip for different inclination angles $\theta$;

b) The shear resistance, computed from the nucelation energy, for differet inclination angles $\theta$. These curves are computed for fcc Ni.

Fourth, we present the critical stress intensity factor $K_{I e}$ observed in the simulations as a function of the slip plane inclination angle as shown in Figure 22a. The analytical predictions for $K_{I e}$ of Eq.15 are also shown, and excellent agreement is obtained except at $\theta=35.3^{\circ}$, where the analytical model is notably larger. The predictions of the Rice model (which works well for $K_{I e}$ in $\mathrm{Ni}$ ) are also shown, and the simulations at $\theta=35.3^{\circ}$ fall below the Rice value as well. The low $K_{I e}$ at $\theta=35.3^{\circ}$ emission is facilitated by the presence of two symmetrical slip systems activated. To demonstrate this, simulations at $\theta=35.3^{\circ}$ were performed by constraining the first structural unit below the crack plane (unit $1^{\mathrm{B}}$; see Figure $22 \mathrm{~b}$ ) to displace according to the elastic K- 
This is a post-print of the following article: Andric, Predrag; Curtin, W. A. Journal Of The Mechanics And Physics Of Solids $2017,23$. 315-337.. The formal publication is available at http://dx.doi.org/10.1016/j.jmps.2017.06.006 @ 2017. This manuscript version is made available under the CC-BY-NC-ND 4.0 license http://creativecommons.org/licenses/by-nc-nd/4.0/

displacement. With this constraint, dislocation emission along the upper slip plane occurs at a much higher $\mathrm{K}_{\mathrm{Ie}}$ that is also in excellent agreement with the prediction of Eq. 15.
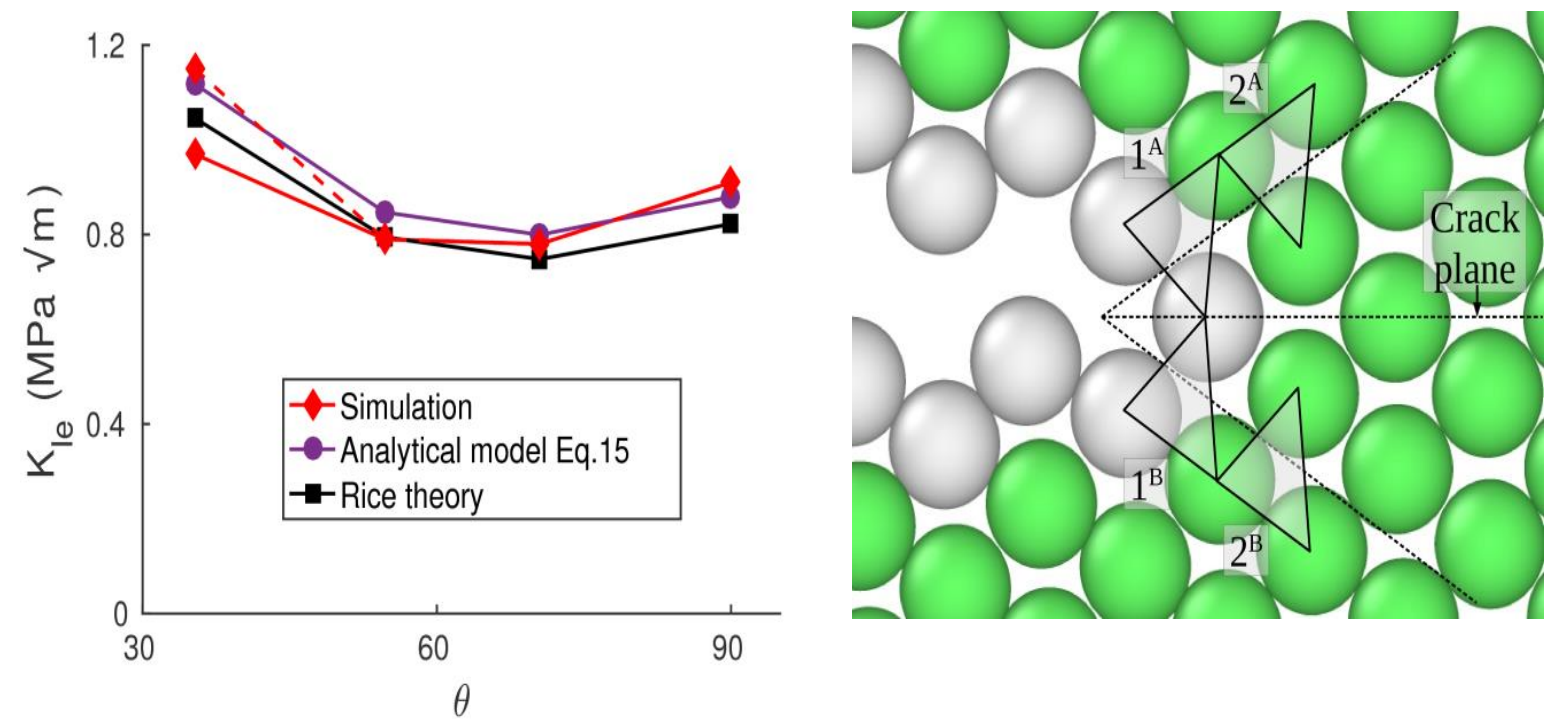

Figure 22: Critical stress intensity factor for dislocation emission as a function of the slip plane inclination angle $\theta$ in fcc Ni; Simulation (red diamonds); Analytical model Eq. 15 (purple circles); Rice theory (black squares); dashed line shows the simulation result when constraining deformation of the symmetric slip plane that exists at the lowest angle. b) Crack tip geometry when $\theta=35.3$ and definition of the structural units along two symmetric slip planes.

We conclude that there are small differences in precise behavior as a function of inclination angle, with somewhat larger deviations if two symmetrical slip systems are activated. And non-linearity and local crack tip geometry will always impart small material-dependent deviations from theoretical idealizations. However, these differences usually have little impact on the quantitative results, and the predictions of the current model remain generally in good agreement with simulations. This enables application of the theory to make good predictions based on computed or experimental material properties (e.g. unstable stacking fault and surface energies) for systems where interatomic potentials do not exist or are inadequate. We also note that the differences shown in Figures 20 and 22 pertain to the Rice theory as well as the present model. The present theory emphasizes the essential role of step creation in determining the major details of dislocation emission, and this important new feature is independent of slip plane inclination angle. 
This is a post-print of the following article: Andric, Predrag; Curtin, W. A. Journal Of The Mechanics And Physics Of Solids $2017,, 23$. 315-337.. The formal publication is available at http://dx.doi.org/10.1016/j.jmps.2017.06.006 @ 2017. This manuscript version is made available under the CC-BY-NC-ND 4.0 license http://creativecommons.org/licenses/by-nc-nd/4.0/

\section{References}

[1] Griffith AA. The phenomena of rupture and flow in solids. Philosophical transactions of the royal society of London. Series A, containing papers of a mathematical or physical character. 1921Jan 1;221:163-98.

[2] Argon AS. Brittle to ductile transition in cleavage fracture. Acta Metallurgica. 1987 Jan 1;35(1):18596.

[3] Armstrong RW. Cleavage crack propagation within crystals by the Griffith mechanism versus a dislocation mechanism. Materials science and engineer.

[4] Kelly A, Tyson WR, Cottrell AH. Ductile and brittle crystals. Philosophical magazine. 1967 Mar 1;15(135):567-86.

[5] Rice JR, Thomson R. Ductile versus brittle behavior of crystals. Philosophical magazine. 1974 Jan 1;29(1):73-97.

[6] Schoeck G. Dislocation emission from crack tips. Philosophical Magazine A. 1991 Jan 1;63(1):111-20.

[7] Rice JR. Dislocation nucleation from a crack tip: an analysis based on the Peierls concept. Journal of the Mechanics and Physics of Solids. 1992 Dec 31;40(2):239-71.

[8] Zhou SJ, Carlsson AE, Thomson R. Dislocation nucleation and crack stability: lattice Green's-function treatment of cracks in a model hexagonal lattice. Physical Review B. 1993 Apr 1;47(13): 7710.

[9] Knap J, Sieradzki K. Crack tip dislocation nucleation in FCC solids. Physical review letters. 1999 Feb 22;82(8):1700.

[10] Gumbsch P, Beltz GE. On the continuum versus atomistic descriptions of dislocation nucleation and cleavage in nickel. Modelling and Simulation in Materials Science and Engineering.1995Sep;3(5):597.

[11] Zhou SJ, Carlsson AE, Thomson R. Crack blunting effects on dislocation emission from cracks. Physical review letters. 1994 Feb 7;72(6):852.

[12] Xu G, Argon AS, Ortiz M. Nucleation of dislocations from crack tips under mixed modes of loading: implications for brittle against ductile behavior of crystals. Philosophical Magazine A. 1995 Aug 1;72(2):415-51.

[13] Juan YM, Sun Y, Kaxiras E. Ledge effects on dislocation emission from a crack tip: a first-principles study for silicon. Philosophical Magazine Letters. 1996 May 1;73(5):233-40.

[14] Schoeck G. The emission of dislocations from crack tips: A critical assessment. Materials Science and Engineering: A. 2003 Sep 15;356(1):93-101.

[15] Sun Y, Beltz GE. Dislocation nucleation from a crack tip: a formulation based on anisotropic elasticity. Journal of the Mechanics and Physics of Solids. 1994 Dec 1;42(12):1905-32.

[16] Stroh AN. Dislocations and cracks in anisotropic elasticity. Philosophical magazine. 1958 Jun $1 ; 3(30): 625-46$.

[17] Hirth JP, Lothe J. Chapter 8.2 - The Peierls-Nabarro Dislocation model, Theory of dislocations. 1982: 218-24.

[18] Curtin WA. On lattice trapping of cracks. Journal of Materials Research. 1990 Jul 1;5(07):1549-60.

[19] Mishin Y, Farkas D, Mehl MJ, Papaconstantopoulos DA. Interatomic potentials for monoatomic metals from experimental data and ab initio calculations. Physical Review B. 1999 Feb 1;59(5):3393.

[20] Ercolessi F, Adams JB. Interatomic potentials from first-principles calculations: the force-matching method. EPL (Europhysics Letters). 1994 Jun 10;26(8):583.

[21] Mishin Y, Mehl MJ, Papaconstantopoulos DA, Voter AF, Kress JD. Structural stability and lattice defects in copper: Ab initio, tight-binding, and embedded-atom calculations. Physical Review B. 2001 May 21;63(22):224106.

[22] Adams JB, Foiles SM, Wolfer WG. Self-diffusion and impurity diffusion of fee metals using the fivefrequency model and the embedded atom method. Journal of Materials Research. 1989 Feb 1;4(01):102-12.

[23] Bonny G, Terentyev D, Pasianot RC, Poncé S, Bakaev A. Interatomic potential to study plasticity in stainless steels: the $\mathrm{FeNiCr}$ model alloy. Modelling and simulation in materials science and engineering. 2011 Nov 3;19(8):085008.

[24] Varvenne C, Luque A, Nöhring WG, Curtin WA. Average-atom interatomic potential for random alloys. Physical Review B. 2016 Mar 11;93(10):104201. 
This is a post-print of the following article: Andric, Predrag; Curtin, W. A. Journal Of The Mechanics And Physics Of Solids $2017,23$. 315-337.. The formal publication is available at http://dx.doi.org/10.1016/j.jmps.2017.06.006 @ 2017. This manuscript version is made available under the CC-BY-NC-ND 4.0 license http://creativecommons.org/licenses/by-nc-nd/4.0/

[25] Plimpton S. Fast parallel algorithms for short-range molecular dynamics. Journal of computational physics. 1995 Mar 1;117(1):1-9.

[26] Stukowski A. Visualization and analysis of atomistic simulation data with OVITO-the Open Visualization Tool. Modelling and Simulation in Materials Science and Engineering. 2009 Dec 15;18(1):015012.

[27] Beltz GE, Lipkin DM, Fischer LL. Role of crack blunting in ductile versus brittle response of crystalline materials. Physical review letters. 1999 May 31;82(22):4468.

[28] Rajan VP, Warner DH, Curtin WA. An interatomic pair potential with tunable intrinsic ductility. Modelling and Simulation in Materials Science and Engineering. 2016 Jan 12;24(2):025005.

[29] Warner DH, Curtin WA. Origins and implications of temperature-dependent activation energy barriers for dislocation nucleation in face-centered cubic metals. Acta Materialia. 2009 Aug 31;57(14):4267-77.

[30] Yamakov VI, Warner DH, Zamora RJ, Saether E, Curtin WA, Glaessgen EH. Investigation of crack tip dislocation emission in aluminum using multiscale molecular dynamics simulation and continuum modeling. Journal of the Mechanics and Physics of Solids. 2014 Apr 30;65:35-53.

[31] Rice JR, Wang JS. Embrittlement of interfaces by solute segregation. Materials Science and Engineering: A. 1989 Jan 1;107:23-40.

[32] Lynch SP. A fractographic study of hydrogen-assisted cracking and liquid-metal embrittlement in nickel. Journal of Materials science. 1986 Feb 1;21(2):692-704.

[33] Zamora RJ, Nair AK, Hennig RG, Warner DH. Ab initio prediction of environmental embrittlement at a crack tip in aluminum. Physical Review B. 2012 Aug 2;86(6):060101.

[34] Wu Z, Curtin WA. Brittle and ductile crack-tip behavior in magnesium. Acta Materialia. $2015 \mathrm{Apr}$ 15;88:1-2.

[35] Sun CT, Jin ZH. Chapter 10 - Special Topics. Fracture mechanics, Academic Press, Boston. 2012: 24753.

[36] Zamora RJ, Baker KL, Warner DH. Illuminating the chemo-mechanics of hydrogen enhanced fatigue crack growth in aluminum alloys. Acta Materialia. 2015 Nov 30;100:232-9. 